\title{
THREE CONSTRUCTIONS OF FROBENIUS MANIFOLDS: A COMPARATIVE STUDY*
}

\author{
YU. I. MANIN ${ }^{\dagger}$
}

\begin{abstract}
The paper studies three classes of Frobenius manifolds: Quantum Cohomology (topological sigma-models), unfolding spaces of singularities (K. Saito's theory, Landau-Ginzburg models), and the recent Barannikov-Kontsevich construction starting with the Dolbeault complex of a Calabi-Yau manifold and conjecturally producing the $B$-side of the Mirror Conjecture in arbitrary dimension. Each known construction provides the relevant Frobenius manifold with an extra structure which can be thought of as a version of "non-linear cohomology". The comparison of these structures sheds some light on the general Mirror Problem: establishing isomorphisms between Frobenius manifolds of different classes. Another theme is the study of tensor products of Frobenius manifolds, corresponding respectively to the Künneth formula in Quantum Cohomology, direct sum of singularities in Saito's theory, and presumably, the tensor product of the differential GerstenhaberBatalin-Vilkovyski algebras. We extend the initial Gepner's construction of mirrors to the context of Frobenius manifolds and formulate the relevant mathematical conjecture.
\end{abstract}

\section{Introduction.}

0.1. Frobenius manifolds. Frobenius manifolds were introduced and investigated by $\mathrm{B}$. Dubrovin as the axiomatization of a part of the rich mathematical structure of the Topological Field Theory (TFT): cf. [D].

According to [D] and [M], a Frobenius manifold is a quadruple $\left(M, \mathcal{T}_{M}^{f}, g, A\right)$. Here $M$ is a supermanifold in one of the standard categories $\left(C^{\infty}\right.$, analytic, algebraic, formal, ...), $\mathcal{T}_{M}^{f}$ is the sheaf of flat vector fields tangent to an affine structure, $g$ is a flat Riemannian metric (non-degenerate even symmetric quadratic tensor) such that $\mathcal{T}_{M}^{f}$ consists of $g$-flat tangent fields. Finally, $A$ is an even symmetric tensor $A: S^{3}\left(\mathcal{T}_{M}\right) \rightarrow \mathcal{O}_{M}$. All these data must satisfy the following conditions;

a) Potentiality of $A$. Everywhere locally there exists a function $\Phi$ such that $A(X, Y, Z)=(X Y Z) \Phi$ for any flat vector fields $X, Y, Z$.

b) Associativity. $A$ and $g$ together define a unique symmetric multiplication $\circ: \mathcal{T}_{M} \otimes \mathcal{T}_{M} \rightarrow \mathcal{T}_{M}$ such that

$$
A(X, Y, Z)=g(X \circ Y, Z)=g(X, Y \circ Z) .
$$

This multiplication must be associative.

In other words, in flat coordinates the tensor of the third derivatives $\Phi_{a b}{ }^{c}$ must constitute the set of structure constants of an associative algebra.

If one excludes the trivial case when $\Phi$ is a cubic form with constant coefficients in flat coordinates, the first large class of Frobenius manifolds was discovered by Kyoji Saito even before Dubrovin's axiomatization (see [S1], [S2] and [O]):

(i) Moduli spaces of unfolding (germs of) isolated singularities of hypersurfaces carry natural structures of Frobenius manifold.

Each such structure is determined by a choice of Saito's good primitive form.

In [D] a more global variation of this construction is described (Hurwitz's spaces).

Physicists call the relevant TFT the topological sector of the Landau-Ginzburg theory: cf. [C].

${ }^{*}$ Received May 23, 1998; accepted for publication March 18, 1999.

† Max Planck Institute for Mathematics, Vivatgasse 7, Bonn 53111, Germany (manin@mpimbonn.mpg.de). 
The second large class of Frobenius manifolds was discovered by physicists (Witten, Dijkgraaf, Vafa) and is called Quantum Cohomology. For an axiomatic treatment, see [KM1]. The correlators of this theory are called Gromov-Witten (GW) invariants. Their actual construction in the algebraic-geometric framework was carried out in [B] following $[\mathrm{BM}]$ and $[\mathrm{BF}]$.

(ii) The formal completion at zero of the cohomology (super) space of any smooth projective or compact symplectic manifold carries a natural structure of formal Frobenius manifold.

The third large class of Frobenius manifolds was recently constructed by S. Barannikov and M. Kontsevich ([BK]).

(iii) The formal moduli spaces of solutions to the Maurer-Cartan equations modulo gauge equivalence, related to a class of the differential Gerstenhaber-BatalinVilkovyski (dGBV) algebras, carry a natural structure of formal Frobenius manifold.

As their main application, Barannikov and Kontsevich construct the dGBValgebra starting with the Dolbeault complex of an arbitrary Calabi-Yau manifold, and conjecture that the resulting formal Frobenius manifold ( $B$-model) can be identified with the quantum cohomology of the mirror dual Calabi-Yau manifold ( $A$-model). S. Merkulov ([Me]) recently invented a similar construction applicable to any symplectic manifold satisfying the strong Lefschetz condition (cf. below, subsections 5.9, 5.10, 6.5 and 6.6). Yet another possible source of dGBV-algebras (or rather their homotopy version) is provided by the BRST cohomology of certain chiral algebras: cf. [LZ].

The Mirror Conjecture is a part of the gradually emerging considerably more general pattern. Within the Calabi-Yau domain, it should be a consequence of the Kontsevich's conjecture about the equivalence of the Fukaya triangulated category associated to one member of the mirror pair and the derived category of sheaves on the other member. Furthermore, one expects the extension of the mirror picture to other classes of varieties, non-necessarily smooth, compact or having trivial canonical class. For some exciting recent results on mirrors, cf. [Giv] and [LLY].

Isomorphisms of Frobenius manifolds of different classes remain the most direct expression, although by no means the final one, of various mirror phenomena.

From this vantage point, the three classes of examples considered above should be compared at least in two ways.

First, one looks for isomorphisms between Frobenius manifolds (and their submanifolds) constructed by different methods.

Second, one tries to generalize to other classes of Frobenius manifolds additional structures peculiar to each of the known classes.

Consider, for example, Quantum Cohomology. Physically, quantum cohomology of a manifold $V$ is only the tree level small phase space part of the topological sigma model with target space $V$. In particular, the correlators of this theory, which are essentially the coefficients of the formal Frobenius potential, can be mathematically defined in terms of the intersection indices on moduli spaces of stable maps of curves of genus zero to $V$. This set of the correlators of Quantum Cohomology of $V$ can be extended to a much vaster structure involving, first, curves of arbitrary genus, and second, the so called gravitational descendants, mathematically expressible via Chern classes of certain tautological bundles on the moduli spaces of stable maps (cf. [KM2] for precise statements). 
This leads to two natural questions.

What is the differential geometric meaning of the arbitrary genus correlators with descendants in the framework of general geometry of Frobenius manifolds?

\section{Which Frobenius manifolds admit extensions of this type?}

Of course, Frobenius manifolds admitting such an extension include those that are isomorphic to Quantum Cohomology. Therefore a better understanding of this problem could shed some light on the Mirror Conjecture as well. In [KM2] it is shown that at least the correlators with gravitational descendants in any genus $g$ can be reconstructed from the additional data consisting of two different parts.

One part is the genus $\leq g$ Cohomological Field Theory in the sense of [KM1] whose correlators take values in the cohomology of the moduli spaces of stable curves with marked points. At the moment it is unclear which abstract Frobenius manifolds can be extended to such genus $\leq g$ geometry and how it can be done.

Another part of the data concerns only genus zero correlators and therefore in principle can be formulated in terms of arbitrary Frobenius manifold. Its existence, however, poses non-trivial restrictions on the manifold which are axiomatized below in the notion of qc-type.

This whole setup can be illuminated by comparison with the motivic philosophy. In principle, any natural structure on the cohomology of an algebraic manifold can be considered as a realization of its motive, and the question which abstract structures of a given type arise from cohomology ("are motivated") is a typical question of the theory of motives.

Quantum Cohomology is a highly nonlinear realization of the motives of smooth projective manifolds. It is functorial, at least in the naive sense, only with respect to isomorphisms. Hence it cannot be extended to the category of the Grothendieck motives in an obvious way. Nevertheless, the natural monoidal structure of motives extends to Frobenius manifolds. Their tensor product in the formal context furnishing the Künneth formula for Quantum Cohomology was constructed in [KM] and [KMK]. R. Kaufmann (cf. [K] and paper in preparation) has shown that the tensor product of convergent potentials converges, and the resulting Frobenius manifold in a sense does not depend on the choice of the base points. This adds some flexibility to the motivic perspective. For example, Frobenius manifolds provide a context in which one can meaningfully speak about cohomology of fractional weight: cf. e.g. the treatment of $A_{n}$-manifolds in 2.3..1 and 3.5 below. It should be also compared with S. Cecotti's suggestion that the TFT's of Landau-Ginzburg type naturally give rise to mixed Hodge structures (see $[\mathrm{C}]$ ).

0.2. Plan of the paper. This paper is dedicated to the study of the Frobenius manifolds of the three classes (i), (ii) and (iii) from the perspective described above.

In $\S 1$ we start with reminding the formalism of Gromov-Witten invariants and the structure of the potential of the Quantum Cohomology. In the treatment of [KM] its terms of degree $\leq 2$ were not fixed because of absence of stable curves of genus zero with $\leq 3$ marked points. However, the Divisor Axiom allows an unambiguous definition of these terms (Proposition 1.3.1). This simple remark is essential for the definition of $\leq 2$ point correlators for abstract formal Frobenius manifolds. We introduce the notion of the manifold of qc-type, which embodies a version of the Divisor Axiom, and show that it allows us to define for such manifolds the correlators with 
gravitational descendants. This is an elaboration of the picture sketched in $\S 3$ of [KM2].

In $\S 2$ we introduce the notion of the (strong) Saito framework. It axiomatizes those properties of the spaces of miniversal deformations of isolated singularities of functions which directly lead to the Frobenius structure. The most difficult and deep aspects of Saito's theory are thereby neatly avoided and become "existence theorems". (This illustrates the advantages of theft in comparison with honest work, as was justly remarked about the axiomatic method in the beginning of this century).

This part is taken from my notes to a lecture course and is included here on suggestion of A. Givental.

The main result of this section is summarized in the formulas (2.12), (2.13) which in the context of Saito's theory refer to the unfolding space of the direct sum of singularities, and in our axiomatic treatment are stated in terms of abstract direct sum diagrams. Another proof of these formulas using oscillating integrals was shown to me by A. Givental.

In $\S 3$ we show first of all, using (2.12) and (2.13), that the direct sum of Saito's frameworks corresponds to the tensor product of the associated Frobenius manifolds.

Looking then at the tensor products $M$ of the Frobenius manifolds $A_{n}$ (deformation space of the singularity $z^{n+1}$ at zero) and more general manifolds with rational spectra we find out that the integral part of their spectra define Frobenius submanifolds $H M$ which look like quantum cohomology of a manifold with trivial canonical class, at least on the level of discrete invariants (cf. below). This argument exactly corresponds to the well known idea of D. Gepner ([Ge1], [Gr], [C]) of building CalabiYau sigma models from the tensor products of minimal models. The numerology is also similar.

Here is an example of our results.

0.2.1. Claim. The manifold $A_{n}^{\otimes n+1}$ contains a (canonically defined) pointed Frobenius submanifold $H A_{n}^{\otimes n+1}$ whose spectrum looks formally like that of evendimensional part of quantum cohomology of an $(n-1)$-dimensional algebraic (or symplectic) manifold $V$.

More precisely, $V$ must have Betti numbers

$$
\begin{aligned}
h^{2 m}(V):= & \text { the number of }\left(i_{1}, \ldots, i_{n+1}\right) \in \mathbf{Z}_{\geq 0}^{n+1}, \text { satisfying } \\
& \frac{1}{m} \sum_{k=1}^{n+1} i_{k}=m(n+1), 0 \leq i_{k} \leq n-1
\end{aligned}
$$

and vanishing (modulo torsion) $c_{1}(V)$.

For example, even Betti numbers must be $(1,19,1)$ for $n=3$, and $(1,101,101,1)$ for $n=4$.

The Poincaré symmetry of them is generally established by the involution $\left(i_{k}\right) \mapsto$ $\left(n-1-i_{k}\right), m \mapsto n-1-m$.

0.2.2. Problem. Is there actually a manifold $V_{n}$ whose Quantum Cohomology contains $H A_{n}^{\otimes n+1}$ ? Is it at least true that $H A_{n}^{\otimes n+1}$ is Frobenius manifold of qc-type? (Notice that $A_{n}$ itself is not of qc-type). 
As was explained above, $A_{n}^{\otimes n+1}$ is the unfolding space at zero of the singularity of $x_{1}^{n+1}+\ldots+x_{n+1}^{n+1}$. An argument which I learned from [C] and (in a different version) from A. Givental then shows that $H A_{n}^{\otimes n+1}$ carries the variation of Hodge structure corresponding to the middle cohomology of the hypersurface $x_{1}^{n+1}+\ldots+x_{n+1}^{n+1}=0$. More precisely, the volume form periods constitute the horizontal sections of one of the structure connections of the Frobenius manifold in question.

Thus Problem 0.2.2 has the flavor of Mirror Conjecture, and of course Gepner's idea was a precursor of the modern studies of the mirrors. Hence at least the case $n=4$ of the Problem 0.2.2 might be reducible to the Givental's treatment of the toric CY threefold (see [Giv] and the subsequent developments due to B.-H. Lian, K. Liu, S.-T. Yau in [LLY]).

The Barannikov-Kontsevich construction $[\mathrm{BK}]$ conjecturally provides another, and quite general, class of Frobenius manifolds of Calabi-Yau $B$-type. To describe it succinctly, notice that the space of vector fields on a Frobenius manifold is simultaneously a Lie (super)algebra and a (super)commutative algebra. The classical example of such combination is the algebra of functions on a Poisson (super)manifold. So it would appear that Frobenius manifolds could come from Poisson structures. However, such a relation cannot be straightforward, because the Poisson identity between the bracket and the multiplication does not hold on Frobenius manifolds. The ingenious twist in [BK] consists in considering instead odd Poisson algebras with two differentials. Under appropriate conditions, the Frobenius manifold structure is then induced on the homology space of such Poisson algebra.

The sections 4-6 of this paper constitute a completely self-contained account of the theory of $[\mathrm{BK}]$ in the axiomatic context of dGBV-algebras. Specifically, in $\S 3$ we supply direct elementary proofs of all results related to the formality and to the structure of Maurer-Cartan moduli spaces at their non-obstructed points. In $\S 4$ we collect a list of basic general properties of dGBV-algebras. Finally, in $\S 6$ we define the relevant formal Frobenius manifolds. We would like to stress the similarity of the formulas defining o-multiplication in the Saito's and Barannikov-Kontsevich constructions: compare (2.3) and (6.1). This supports the expectation that both construction might be special cases of a more general picture.

As a comment to the title of Cecotti's paper [C], it is instructive to compare the extensions of the Calabi-Yau variations of Hodge structure (VHS) given by Saito's theory to the construction of [BK] and the equivariant theory of Givental ([Giv]).

Barannikov and Kontsevich embed any Calabi-Yau VHS into a Frobenius supermanifold that has all discrete invariants perfectly matching those of the mirror dual quantum cohomology. Thus it has a good chance to be the correct $B$-model in the classical Mirror Conjecture picture. A drawback of this embedding is that the relevant Frobenius manifolds are not semisimple, and so the identification of them hardly can be achieved by formal calculations.

To the contrary, whenever methods of Gepner, Saito and Givental are applicable (quasi-homogeneous singularities, anticanonical hypersurfaces in toric compactifications), they embed (parts of) Calabi-Yau VHS into Frobenius manifolds that are generically semisimple or into families of manifolds with generically semisimple general fiber. This makes more accessible the direct check of mirror isomorphisms (cf. $\S 3$ below). But these techniques do not give the full dimension spectrum (e.g. odddimensional cohomology is skipped) and they are not directly applicable to those 
Calabi-Yau manifolds which are not toric anticanonical hypersurfaces (or complete intersections).

ACKNOWLEDGEMENTS. The first draft of this paper was written after stimulating discussions of Gepner's work with V. Schechtman. It was read and extensively commented by A. Givental who corrected some mistakes and supplied illuminating explanations of the Saito theory. In particular, the whole $\S 2$ owes its existence to Givental's suggestion. In writing the last three sections, I have greatly benefited from M. Kontsevich's lectures at the MPI and his handwritten notes.

\section{Quantum cohomology and Frobenius manifolds.}

1.1. Gromov-Witten (GW) invariants. We start with reminding some basic notation and facts from [KM1], [BM], [B].

Let $V$ be a smooth projective algebraic manifold over an algebraically closed field of characteristic zero, $B=B(V)$ the semigroup of effective one-dimensional algebraic cycles modulo numerical equivalence. For any $\beta \in B(V), g, n \geq 0$ we can define the Deligne--Mumford stack $\bar{M}_{g, n}(V, \beta)$ parametrizing stable maps of curves of genus $g$ with $n$ labelled points, landing in class $\beta$. This stack comes equipped with virtual fundamental class in the homological Chow group with rational coefficients $J_{g, n}(V, \beta) \in A_{s}\left(\bar{M}_{0, n}(V, \beta)\right)$ where

$$
s=(1-g)(\operatorname{dim} V-3)+\left(c_{1}(V), \beta\right)+n .
$$

Moreover, there are canonical morphisms $e v: \bar{M}_{g, n}(V, \beta) \rightarrow V^{n}$ sending a stable map to the image of the family of labelled points. In the stable range, that is when $2 g-2+n>0$, there is also a map $s t: \bar{M}_{g, n}(V, \beta) \rightarrow \bar{M}_{g, n}$ forgetting $V$ and stabilizing the curve. They can be used in order to define the Gromov-Witten correspondences in the Chow rings

$$
I_{g, n}(V, \beta):=(e v, s t)_{*}\left(J_{g, n}(V, \beta)\right) \in A_{s}\left(V^{n} \times \bar{M}_{g, n}\right) .
$$

This family of Chow correspondences is the most manageable embodiment of motivic quantum cohomology forgetting just the right amount of geometric information encoded in the rather uncontrollable stack of stable maps. For genus zero, the situation further simplifies. Since $V^{n}$ and $\bar{M}_{0, n}$ are smooth, we can identify $A_{*}$ with $A^{*}$. As S. Keel proved, $A^{*}\left(V^{n} \times \bar{M}_{0, n}\right)=A^{*}\left(V^{n}\right) \otimes A^{*}\left(\bar{M}_{0, n}\right)$, and $A^{*}\left(\bar{M}_{0, n}\right)$ is a finitedimensional self-dual linear space. Hence one can identify $I_{0, n}(V, \beta)$ with the induced map $A^{*}\left(\bar{M}_{0, n}\right) \rightarrow A^{*}\left(V^{n}\right)$. The space $A^{*}\left(\bar{M}_{0, n}\right)$ is spanned by the dual classes of the boundary strata $\bar{M}(\tau)$ indexed by $n$-trees. So calculating $I_{0, n}(V, \beta)$ amounts to calculating a finite family of elements in $A^{*}\left(V^{n}\right)$ indexed by these trees.

1.2. Frobenius manifolds. All our examples of Frobenius manifolds (see 0.1) will come equipped with two additional structures: a flat vector field $e$ which is identity with respect to $\circ$, and an Euler vector field $E$ expressing the scaling invariance of the Frobenius manifold $M$. More precisely, we must have $\operatorname{Lie}_{E}(g)=D g$ for some constant $D$, and $\operatorname{Lie}_{E}(\mathrm{o})=d_{0} \circ$ for another constant $d_{0}$ (in the context of Frobenius manifolds $g$ means the metric, not the genus, which is zero for the relevant $\mathrm{GW}$-invariants). If the first condition is satisfied, the second one is equivalent to

$$
E \Phi=\left(d_{0}+D\right) \Phi+\text { a polynomial in flat coordinates of degree } \leq 2 .
$$

For any Euler field $E$ we have $\left[E, \mathcal{T}_{M}^{f}\right] \subset \mathcal{T}_{M}^{f}$. Assume for simplicity that the 
spectrum $\left\{d_{a}\right\}$ of $-\operatorname{ad} E$ on flat vector fields belongs to the base field. We understand $\left\{d_{a}\right\}$ as a family of constants with multiplicities. The constant $d_{0}$ introduced earlier is in addition the eigenvalue corresponding to $e$. The family $D,\left\{d_{a}\right\}$ is called the spectrum of $M$. Since any multiple of $E$ is an Euler field together with $E$, in the case $d_{0} \neq 0$ we can normalize $E$ by the condition $d_{0}=1$.

We also put $d=2-D$ and $q_{a}=1-d_{a}$ and call the family $d,\left\{q_{a}\right\}$ the $d$-spectrum of $M$.

If $M$ is the formal spectrum of the ring of formal series in flat coordinates, $\Phi$ is a formal solution of the Associativity Equations, we call $M$ formal Frobenius manifold. Formal Frobenius manifolds can be tensor multiplied. The underlying metric space of flat fields of the tensor product is the usual tensor product of the respective spaces of factors. The potential of the tensor product is defined in a much subtler way: see [KM1] and [M]. If the factors are additionally endowed with flat identities and normalized Euler fields, they can be used to produce a canonical flat identity and Euler field on the tensor product: see $[\mathrm{K}]$.

1.3. From genus zero GW invariants to Frobenius manifolds. In the situation of 1.1 , we can construct a formal Frobenius manifold $H_{\text {quant }}^{*}(V)$ whose underlying linear supermanifold is the completion of $H^{*}(V, \Lambda)$ at zero, with obvious flat structure and Poincaré form as metric. Here $\Lambda$ is a $\mathrm{Q}$-algebra endowed with the universal character $B(V) \rightarrow \Lambda: \beta \mapsto q^{\beta}$, with values in the Novikov ring $\Lambda$ which is the completed semigroup ring of $B(V)$ eventually localized with respect to the multiplicative system $q^{\beta}$. It is topologically spanned by the monomials $q^{\beta}=q_{1}^{\beta_{1}} \ldots q_{m}^{b_{m}}$ where $\beta=\left(b_{1}, \ldots, b_{m}\right)$ in a basis of the numerical class group of 1-cycles, and $\left(q_{1}, \ldots, q_{m}\right)$ are independent formal variables. This is needed to make convergent the formal sums

$$
I_{g, n}(V):=\sum_{\beta \in B} q^{\beta} I_{g, n}(V, \beta) \in A_{\Lambda}^{*}\left(V^{n} \times \bar{M}_{g, n}\right)
$$

For $H^{*}$ we can take any cohomology theory functorial with respect to Chow correspondences. The construction of the potential requires only the top degree terms of the genus zero GW invariants. To be concrete, choose a homogeneous basis $\left\{\Delta_{a} \mid a=\right.$ $0, \ldots r\}$ of $H^{*}(V, \mathbf{Q})$. Denote by $\left\{x_{a}\right\}$ the dual coordinates and by $\Gamma=\sum_{a} x_{a} \Delta_{a}$ the generic even element of the cohomology superspace. This means that $\mathbf{Z}_{2}$-parity of $x_{a}$ equals 0 (resp. 1), if $\Delta_{a}$ is even-dimensional (resp. odd-dimensional). Put for $\gamma_{i} \in H^{*}(V)$

$$
\left\langle I_{g, n}(V)\right\rangle\left(\gamma_{1} \otimes \cdots \otimes \gamma_{n}\right)=\left\langle\gamma_{1} \ldots \gamma_{n}\right\rangle_{g, n}:=\int_{I_{g, n}(V)} p r^{*}\left(\gamma_{1} \otimes \cdots \otimes \gamma_{n}\right)
$$

where $p r: V^{n} \times \bar{M}_{g, n} \rightarrow V^{n}$ is the projection. Then the quantum cohomology potential is

$$
\Phi(x)=\left\langle e^{\Gamma}\right\rangle_{0}:=\sum_{n,\left(a_{1}, \ldots, a_{n}\right)} \epsilon(a) \frac{x_{a_{1}} \ldots x_{a_{n}}}{n !}\left\langle\Delta_{a_{1}} \ldots \Delta_{a_{n}}\right\rangle_{0, n}
$$

where $\epsilon(a)$ is the sign resulting from rewriting $\prod x_{i} \Delta_{a_{i}}$ as $\epsilon(a) \prod x_{i} \prod \Delta_{a_{i}}$. Assume that the dual fundamental class $\Delta_{0}$ of $V$ is the part of our basis. Then the flat identity is $\partial_{0}=\partial / \partial x_{0}$. Moreover, the Euler field is

$$
E=\sum_{a}\left(1-\frac{\left|\Delta_{a}\right|}{2}\right) x^{a} \partial_{a}+\sum_{b:\left|\Delta_{b}\right|=2} r^{b} \partial_{b}
$$


where $\Delta_{a} \in H^{\left|\Delta_{a}\right|}(V)$, and $r^{b}$ are defined by

$$
c_{1}\left(\mathcal{T}_{V}\right)=-K_{V}=\sum_{b:\left|\Delta_{b}\right|=2} r^{b} \Delta_{b}
$$

Clearly, $-\operatorname{ad} E$ is semisimple on flat vector fields. Let $H\left(d_{a}\right)$ be the eigenspace correspondng to $d_{a}$. We have $H\left(d_{a}\right)=H^{2 q_{a}}(V)$. Hence the total spectrum is

$$
D=2-\operatorname{dim} V, d_{0}=1, d_{a}=1-\frac{\left|\Delta_{a}\right|}{2} \text { of multiplicity } \operatorname{dim} H^{\left|\Delta_{a}\right|}(V)
$$

and the $d$-spectrum is

$$
d=\operatorname{dim} V,\left\{q \text { of multiplicity } h^{2 q}(V)\right\} .
$$

Quantum cohomology of the direct product of manifolds is the tensor product of the respective Frobenius manifolds. So the $d$-spectrum behaves as is expected.

$E$ itself is not flat, but it has the flat projection $E(0)$ to $H(D)=H^{2}$ which is just the anticanonical class, if $E$ is normalized as above by $d_{0}=1$ and $H^{*}(V)$ is identified with the space of flat vector fields. This is evident from (1.3) and (1.4).

The spaces $H\left(d_{a}\right), H\left(d_{b}\right)$ are orthogonal unless $d_{a}+d_{b}=D$, or equivalently $q_{a}+q_{b}=d$, and dual in the latter case. They also all have integral structure compatible with metric. If we work with a coefficient ring $\Lambda$, these subspaces are direct (free) sumbodules. Identity belongs to $H(1)=H^{0}(V)$. Especially important are $H^{2}(V)=$ $H(D), H_{2}(V)=H(0)$, and the semigroup $B \subset H_{2, \mathbf{Z}}$ of effective algebraic classes, in which every element is finitely decomposable, and zero is indecomposable. They are never trivial for projective smooth $V$ of positive dimension.

Returning to the potential $\Phi$, we see that since $\bar{M}_{0, n}$ is empty for $n \leq 2$, the definition (1.3) specifies only its terms of degree $\geq 3$ in $x_{a}$. The validity of the Associativity Equations is not sensitive to this indeterminacy. However, the missing terms can be uniquely normalized either geometrically, by integrating over $J_{0, n}(V, \beta)$ in $\bar{M}_{0, n}(V, \beta)$ for $n \leq 2$, or formally, by using the Divisor Axiom of [KM1]. Since this normalization is important for the future use, we describe it explicitly. Denote by $\delta:=\sum_{a: d_{a}=0} x_{a} \Delta_{a}$ the generic even element of $H^{2}$.

1.3.1. Proposition. There exists a unique formal function $\Phi$ differing from (1.3) only by terms of degree $\leq 2$ which is representable as a formal Fourier series in $q^{\beta} e^{(\beta, \delta)}, \beta \in B$, with coefficients which are formal series of the remaining coordinates, having the following properties. Put $\Phi=\Psi+c$ where $c$ is the constant $(\beta=0)$ term of the Fourier series. Then, assuming $d_{0}=1$ and denoting by $E(0)$ the anticanonical class summand of $E$, we have:

a) $E \Psi=(D+1) \Psi$.

b) $c$ is a cubic form with $(E-E(0)) c=(D+1) c$, the classical cubic selfintersection index divided by 6.

In fact, if one puts formally $q^{\beta}=0$ for $\beta \neq 0$ in the structure constants of the quantum multiplication, one gets the classical cup multiplication on $H^{*}(V)$ which together with metric determines $c$ starting even with non necessarily normalized potential.

Proof. We use the properties of $I_{0, n}(V, \beta)$ stated axiomatically in [KM1] and proved in $[\mathrm{B}]$. 
The initial potential $\Phi$ is expressed via Gromov-Witten invariants

$$
\Phi(\Gamma):=\sum_{n \geq 3} \sum_{\beta} \frac{q^{\beta}}{n !}\left\langle I_{0, n}(V, \beta)\right\rangle\left(\Gamma^{\otimes n}\right)
$$

where $\left\langle I_{0, n}(V, \beta)\right\rangle$ is defined by the same formula as (1.2) only with integral taken over $I_{0, n}(V, \beta)$. The part of $\Phi$ corresponding to $\beta=0$ is exactly $c=\frac{1}{6}\left(\Gamma^{3}\right)$.

The maps $\left\langle I_{0, n}(V, \beta)\right\rangle: H^{*}(V)^{\otimes n} \rightarrow \Lambda$ are defined for $n \geq 3$ and satisfy

$$
\left\langle I_{0, n}(V, \beta)\right\rangle(\alpha \otimes \delta)=(\beta, \delta)\left\langle I_{0, n-1}(V, \beta)\right\rangle(\alpha)
$$

for $\delta \in H^{2}(V)$ (we write $(\beta, \delta)$ for $g(\beta, \delta)$ ). This follows from the Divisor Axiom. It is easy to check that there exists a unique polylinear extension of $\left\langle I_{0, n}(V, \beta)\right\rangle$ to all $n \geq 0$ satisfying this identity. In fact, it suffices to put

$$
\left\langle I_{0, n}(V, \beta)\right\rangle(\alpha)=(\beta, \delta)^{-m}\left\langle I_{0, n+m}(V, \beta)\right\rangle\left(\alpha \otimes \delta^{\otimes m}\right)
$$

for any $(m, \delta)$ with $m+n \geq 3$ and invertible $(\beta, \delta)$.

Now put

$$
\Psi(\Gamma):=\sum_{n \geq 0} \sum_{\beta \neq 0} \frac{q^{\beta}}{n !}\left\langle I_{0, n}(V, \beta)\right\rangle\left(\Gamma^{\otimes n}\right)
$$

Clearly, $\Psi+c$ differs from the initial $\Phi$ by terms of degree $\leq 2$. Moreover, $c$ is a linear combination of $x^{a} x^{b} x^{c}$ with $\left|\Delta_{a}\right|+\left|\Delta_{b}\right|+\left|\Delta_{c}\right|=2 \operatorname{dim} V$ so that $(E-E(0)) c=(D+1) c$. As for $E \Psi$, we have for $\Gamma=\gamma_{0}+\delta$

$$
\begin{gathered}
\Psi\left(\gamma_{0}+\delta\right)=\sum_{i, k \geq 0} \sum_{\beta \neq 0} \frac{q^{\beta}}{i ! k !}\left\langle I_{0, n}(V, \beta)\right\rangle\left(\gamma_{0}^{\otimes i} \otimes \delta^{\otimes k}\right)= \\
\sum_{i \geq 0} \sum_{\beta \neq 0} \frac{q^{\beta} e^{(\beta, \delta)}}{i !}\left\langle I_{0, n}(V, \beta)\right\rangle\left(\gamma_{0}^{\otimes i}\right) .
\end{gathered}
$$

Let us apply now $E$ to any summand in (1.8). The $E(0)$ part acts only upon $e^{(\beta, \delta)}$ and multiplies it by $\left(c_{1}(V), \beta\right)$. The $E-E(0)$ part multiplies any monomial $x_{a_{1}} \ldots x_{a_{n}}$ in non-divisorial coordinates by $\sum_{i}\left(1-\left|\Delta_{a_{i}}\right| / 2\right)$. From (1.2) and (1.3) we see that $\beta$ can furnish a non-zero contribution to such term only if

$$
\operatorname{dim} I_{g, n}(V, \beta)=\operatorname{dim} V-3+\left(c_{1}(V), \beta\right)+n=\sum_{i=1}^{n} \frac{\left|\Delta_{a_{i}}\right|}{2} .
$$

Hence every non-vanishing term of (1.8) is an eigenvector of $E$ with eigenvalue $D+1=$ $3-\operatorname{dim} V$.

This proves the Proposition.

Notice in conclusion that $q^{\beta} e^{(\beta, \delta)}$ is the universal character of $B$ together with $q^{\beta}$. We have introduced $q^{\beta}$ only to achieve the formal convergence. If it holds without $q^{\beta}$, we can forget about it. Moreover, if the formal Fourier series actually converges for $\delta$ lying somewhere in the complexified ample cone, $\Psi(x)$ has a free abelian symmetry group: translations by an appropriate discrete subgroup in the space $H^{2}(V, i \mathbf{R})$. Conversely, in the analytic category this condition is necessary for the existence of the appropriate Fourier series. 
1.4. Potentials of qc-type. Based upon the analysis above, we will introduce the following definition. Its first goal is to axiomatize a part of the structures of sec. 1.3 which suffices for the construction of the coupling of a formal Frobenius manifold with gravity in the sense of [KoM2]. As we will recall below, this construction is divided into two steps: the construction of the modified gravitational descendants which can be done for any formal Frobenius manifold, and the construction of a linear operator $T$ on the big phase space which requires additional structures.

The second goal is to provide an intermediate step in the problem of checking whether a given formal Frobenius manifold is quantum cohomology. We must be able at least to detect the following structures.

1.4.1. Definition. Let $\left(M=\operatorname{Spf} k\left[\left[H^{t}\right]\right], g, \Phi_{0}\right)$ be a formal Frobenius manifold over a Q-algebra $k$ with flat identity, Euler field $E$, and spectrum $D,\left\{d_{a}\right\}$ in $k$ as above. Here $H$ is a free $\mathbf{Z}_{2}$-graded $k$-module of flat vector fields, and $H^{t}$ is the dual module of flat coordinates vanishing at the origin. Put $H^{2}=H(0), H_{2}=H(D)$.

Assume that there exists a semigroup $\mathrm{B} \subset \mathrm{H}_{2}$ with finite decomposition and indecomposable zero, and the cubic form $c$ on $H$, such that by eventually changing terms of degree $\leq 2$ in $\Phi_{0}$ we can obtain the potential of the form

$$
\begin{gathered}
\Phi=\Psi+c, E \Psi=(D+1) \Psi,(E-E(0)) c=(D+1) c, \\
\Psi\left(\gamma_{0}+\delta\right)=\sum_{i \geq 0} \sum_{\beta \in B \backslash\{0\}} \frac{e^{(\beta, \delta)}}{i !} I_{\beta}\left(\gamma_{0}^{\otimes i}\right)
\end{gathered}
$$

such that all summands in the last sum are eigenvectors of $E$ with eigenvalue $D+1$. Here $\gamma$ is a generic even element of $H, \delta$ its "divisorial" $H^{2}-$ part, $\gamma_{0}=\gamma-\delta$. The coefficient $I_{\beta}\left(\gamma_{0}^{\otimes i}\right)$ is a form in non-divisorial coordinates.

A formal Frobenius manifold satisfying these conditions will be called of qc-type.

A flat identity $e$ in this language is an element $e \in H$ which considered as a derivation satisfies

$$
e \Psi=0, e c=g
$$

(see $[\mathrm{M}]$, p. 29 for the same expressions in coordinates).

1.4.2. Correlators of qc-manifolds. Let $M$ be a formal Frobenius manifold of qc-type. Recall that $\Phi_{a b}^{c}$ are the structure constants of the quantum multiplicarion. On qc-manifolds there are two useful specializations of this structure.

a) The "small quantum multiplication" obtained by restricting $\Phi_{a b}^{c}$ to $\gamma_{0}=0$. We will denote this multiplication by dot.

b) The cup multiplication $\cup$ obtained by putting formally $e^{(\beta, \gamma)}=0$ for all $\beta \neq 0$ ("large volume limit"). In other words, this is the multiplication, for which $c$ can be written as

$$
c(\gamma)=\frac{1}{6} g(\gamma, \gamma \cup \gamma)
$$

We now define correlators $\langle\ldots\rangle: H^{\otimes n} \rightarrow k$ as $S_{n}$-invariant polylinear functions whose values are derivatives of $\Phi$ at zero. In other words, for a basis $\left\{\Delta_{a}\right\}$ of $H$ and dual coordinates $\left\{x_{a}\right\}$ as above, we have

$$
\Phi(x)=\sum_{n, a_{1}, \ldots, a_{n}} \epsilon(a) \frac{x_{a_{1}} \ldots x_{a_{n}}}{n !}\left\langle\Delta_{a_{1}} \ldots \Delta_{a_{n}}\right\rangle
$$


In the qc-case we can write

$$
\left\langle\Delta_{a_{1}} \ldots \Delta_{a_{n}}\right\rangle=\sum_{\beta \in B \backslash\{0\}}\left\langle\Delta_{a_{1}} \ldots \Delta_{a_{n}}\right\rangle_{\beta}+\left\langle\Delta_{a_{1}} \ldots \Delta_{a_{n}}\right\rangle_{0}
$$

where the first sum comes from $\Psi$ and the second, nonvanishing only for triple arguments, from $c$.

Looking at (1.9) one sees, that small quantum multiplication depends only on the triple correlators of non-divisorial elements of the basis.

1.4.3. Claim. The correlators of the Frobenius manifolds of qc-type satisfy the following Divisor Identity: if $\delta \in H^{2}, \beta \neq 0$,

$$
\left\langle\delta \gamma_{1} \ldots \gamma_{n}\right\rangle_{\beta}=(\delta, \beta)\left\langle\gamma_{1} \ldots \gamma_{n}\right\rangle_{\beta}
$$

Reading backwards the proof of (1.8), one sees that this property follows from (1.9).

This formula allows us to extend the definition of the correlators to $n \leq 2$ arguments.

1.5. Gravitational descendants for the Frobenius manifolds of qctype. Let now $M$ be a formal Frobenius manifold as in 1.4.1 whose space we identify with the linear superspace $H$, At first we do not assume that it is of qc-type. Following [KM2], we can define its modified correlators with gravitational descendants. They are polylinear functions on the big phase space $\oplus_{d \geq 0} H[d]$ where $H[d]$ are copies of the space $H$ identified with the help of the shift operator $\tau: H[d] \rightarrow H[d+1]$. To define them explicitly, we recall that any formal Frobenius manifold gives rise to the genus zero Cohomological Field Theory. Namely, there exists a unique sequence of linear maps $I_{n}^{M}: H^{\otimes n} \rightarrow H^{*}\left(\bar{M}_{0, n}, k\right), n \geq 3$, satisfying the folowing properties.

a) $I_{n}^{M}$ are $S_{n}$-invariant and compatible with restriction to the boundary divisors (cf. [KM1] or [M], p. 101).

b) The top degree term of $I_{n}^{M}$ capped with the fundamental class of $\bar{M}_{0, n}$ is the correlator of $M$ with $n$ arguments.

Moreover, in the quantum cohomology case

$$
I_{n}^{M}=\sum_{\beta} q^{\beta} I_{0, n, \beta}^{V}
$$

where $I_{0, n, \beta}^{V}$ are the genus zero Gromov-Witten invariants.

Now let $C \rightarrow \bar{M}_{0, n}$ be the universal curve, $s_{i}: \bar{M}_{0, n} \rightarrow C, i=1, \ldots, n$ its structure sections, $\omega_{C}$ the relative dualizing sheaf, $\psi_{i}:=c_{1}\left(s_{i}^{*}\left(\omega_{c}\right)\right) \in A^{1}\left(\bar{M}_{0, n}\right.$. The the modified correlators with gravitational descendants for $M$ are defined by the formula

$$
\left\langle\tau^{d_{1}} \Delta_{a_{1}} \ldots \tau^{d_{n}} \Delta_{a_{n}}\right\rangle:=\int_{\bar{M}_{0, n}} I_{n}^{M}\left(\Delta_{a_{1}} \otimes \cdots \otimes \Delta_{a_{n}}\right) \psi_{1}^{d_{1}} \ldots \psi_{n}^{d_{n}}
$$

and the generator function for them, the modified potential, by the formula

$$
G^{M}(x)=\sum_{n \geq 3,\left(a_{i}, d_{i}\right)} \epsilon(a) \frac{x_{d_{1}, a_{1}} \ldots x_{d_{n}, a_{n}}}{n !}\left\langle\tau^{d_{1}} \Delta_{a_{1}} \ldots \tau^{d_{n}} \Delta_{a_{n}}\right\rangle
$$

where $\left(x_{d, a}\right)$ are coordinates dual to $\tau^{d} \Delta_{a}$. 
If we assume in addition that $M$ is of qc-type, we can define also the (unmodified) two argument correlators $\left\langle\tau_{d} \gamma_{1} \gamma_{2}\right\rangle$ by the inductive formula (25) from [KM2]:

$$
\begin{gathered}
\left\langle\tau_{d} \gamma_{1} \gamma_{2}\right\rangle=\sum_{j=1}^{d}(-1)^{j+1} \partial_{\delta}^{-j}\left\langle\tau_{d-j} \gamma_{1} \delta \cdot\left(\delta^{j-1} \cup \gamma_{2}\right)\right\rangle+ \\
(-1)^{d} \partial_{\gamma_{0}}^{-(d+1)}\left[\left\langle\delta \gamma_{1} \delta^{d} \cup \gamma_{2}\right\rangle-\left\langle\delta \gamma_{1} \delta^{d} \cup \gamma_{2}\right\rangle_{0}\right] .
\end{gathered}
$$

Here $\delta \in H^{2}$ is an arbitrary (say, generic) element such that $(\delta, \beta) \neq 0$ for all $\beta \in$ $B \backslash\{0\}$ and the operator $\partial_{\delta}^{-1}$ divides $\langle\ldots\rangle_{\beta}$ by $(\delta, \beta)$.

Furthermore, put

$$
y_{c, b}=x_{c, b}+\sum_{(a, d), g \geq c+1} x_{d, a}\left\langle\tau_{d-c-1} \Delta_{a} \Delta^{b}\right\rangle
$$

Then the big phase space potential of $M$ is, by definition, $F^{M}(x):=G^{M}(y)$, and the unmodified correlators with gravitational descendants of $M$ are defined as coefficients of $F$ :

$$
F^{M}(x)=\sum_{n \geq 3,\left(a_{i}, d_{i}\right)} \epsilon(a) \frac{x_{d_{1}, a_{1}} \ldots x_{d_{n}, a_{n}}}{n !}\left\langle\tau_{d_{1}} \Delta_{a_{1}} \ldots \tau_{d_{n}} \Delta_{a_{n}}\right\rangle
$$

The main result of [KM2] is that if $M$ is the quantum cohomology of $V$, this prescription provides the correlators with descendants of the topological sigma model with target space $V$. The latter are defined by the formula similar to (1.10) but with $\bar{M}_{0, n}$ replaced by $\bar{M}_{0, n}(V), I$ replaced by $J$, and the respective change in the meaning of $\psi_{i}$

In conclusion notice that the sigma model correlators satisfy, partly demonstrably, partly conjecturally, some additional identities, of which the most interesting are probably the Virasoro constraints. I do not know which of these identities might be valid for the more general qc-type manifolds. In any case, it would be interesting to determine differential equations at least for the modified potential with descendants for general formal Frobenius manifolds or particular examples like the manifolds $A_{n}$ (see 2.3. below).

\section{K. Saito's frameworks.}

2.1. Setup. Let $p: N \rightarrow M$ be a submersion of complex analytic or algebraic manifolds, generally non-compact, $F$ a holomorphic function on $N$. We consider $F$ as a family of functions on the fibers of $p$ parametrized by points of $M$. In local coordinates $z=\left(z_{a}\right), t=\left(t_{b}\right)$ where $t_{b}$ are constant along the fibers of $p$ we write $F=F(z, t)$.

Let $d_{p}: \mathcal{O}_{N} \rightarrow \Omega_{N / M}^{1}$ be the relative differential. Denote by $C$ the closed analytic subspace (or subscheme) of the critical points of the restrictions of $F$ to the fibers given by the equation $d_{p} F=0$. Its ideal $J_{F}$ is locally generated by the partial derivatives $X F$ where $X$ are vertical vector fields on $N$. Derivatives $\partial F / \partial z_{a}$ of course suffice. Let $i_{C}: C \rightarrow N$ be the natural embedding, $p_{C}$ the restriction of $p$ to $C$.

Denote by $\Omega_{N / M}^{\max }$ the invertible sheaf of holomorphic vertical volume forms on $N$, $L:=i_{C}^{*}\left(\Omega_{N / M}^{\max }\right)$. The Hessian Hess $(F) \in \Gamma\left(C, L^{2}\right)$ is a well defined section of $L^{2}$ which 
in local coordinates as above can be written as

$$
\operatorname{Hess}(F)=i_{C}^{*}\left[\operatorname{det}\left(\frac{\partial^{2} F}{\partial z_{a} \partial z_{b}}\right)\left(d z_{1} \wedge \cdots \wedge d z_{n}\right)^{2}\right] .
$$

We denote by $G_{C} \subset C$ the subspace Hess $(F)=0$. Let $\mathcal{T}_{M}$ be the tangent sheaf of $M$. Finally, let $\omega$ be a nowhere vanishing global section of $\Omega_{N / M}^{\max }$.

2.1.1. Definition. The family $(p: N \rightarrow M, F, \omega)$ is called Saito's framework, if the following conditions are satisfied:

a) Let the map $s: \mathcal{T}_{M} \rightarrow p_{C *}\left(\mathcal{O}_{C}\right)$ be defined by $X \mapsto \bar{X} F \bmod J_{F}$, where $\bar{X}$ is any local (in $N$ ) lift of $X$. Then $s$ is an isomorphism of $\mathcal{O}_{M}-$ modules. In particular, $C$ is finite and flat over $M$. Assume moreover that $G_{C}$ is a divisor, and $p_{C}: C \rightarrow M$ is étale on the complement to the divisor $G=i_{C *}\left(G_{C}\right) \subset M$.

b) Define the following 1-form $\epsilon$ on $M \backslash G$. Its value on the vector field $X=s^{-1}(f)$ corresponding to the local section $f$ of $\pi_{*}\left(\mathcal{O}_{C}\right)$ equals

$$
i_{X}(\epsilon):=\operatorname{Tr}_{C / M}\left(\frac{f i_{C}^{*}\left(\omega^{2}\right)}{\operatorname{Hess}(F)}\right)=\sum_{i=1}^{\mu} \frac{f\left(\rho_{i}\right)}{\operatorname{det}\left(\left(\partial^{2} F / \partial z_{a} \partial z_{b}\right)\left(\rho_{i}\right)\right)}
$$

where $\rho_{i}$ are the local branches of the critical locus $C$ over $M,\left(z_{a}\right)$ is any vertical local coordinate system unimodular with respect to $\omega$.

Then the scalar product $g: S^{2}\left(\mathcal{T}_{M \backslash G}\right) \rightarrow \mathcal{O}_{M \backslash G}$ defined by

$$
g(X, Y):=i_{X \circ Y}(\epsilon)
$$

is a flat metric. Both $\epsilon$ and $g$ (as flat metric) extend regularly to $M$.

2.2. The (pre-)Frobenius structure associated to the Saito framework. Let $(p: N \rightarrow M, F, \omega)$ be a Saito framework.

Denote by $\circ$ the multiplication in $\mathcal{T}_{M}$ induced by the one in $p_{*}\left(\mathcal{O}_{C}\right)$ :

$$
X \circ Y:=s^{-1}(\bar{X} F \cdot \bar{Y} F) \bmod J_{F}
$$

or equivalently

$$
\overline{X \circ Y} F \equiv \bar{X} F \cdot \bar{Y} F \bmod J_{F} .
$$

Clearly, the vector field $e:=s^{-1}\left(1 \bmod J_{F}\right)$ is the identity for $\circ$. Let $\mathcal{T}_{M}^{f}$ be the sheaf of vector fields flat with respect to $g$. Finally, put

$$
A(X, Y, Z)=g(X \circ Y, Z)=g(X, Y \circ Z) .
$$

The last equality follows from (2.2) and the associativity of $\circ$. The tensor $A$ is symmetric because $\circ$ is commutative. Therefore we have:

2.2.1. Claim. The data $\left(\mathcal{T}_{M}^{f}, g, A\right)$ define on $M$ the structure of pre-Frobenius manifold in the sense of [M], Ch. I, Def. 1.1.1.

One can say more about this structure restricted to $M \backslash G$. Call a connected open subset $U$ in $M \backslash G$ small if $p_{C}^{-1}(U)$ is the disjoint union of $\mu=\operatorname{dim} M$ connected components $U_{i}$ canonically isomorphic to $U$. For concreteness, we will arbitrarily number them by $\{1, \ldots, \mu\}$ as in (2.1). Then we have natural ring isomorphisms

$$
\Gamma\left(U, p_{C *}\left(\mathcal{O}_{C}\right)\right)=\oplus_{i=1}^{\mu} \Gamma\left(U_{i}, \mathcal{O}_{C}\right)=\Gamma\left(U, \mathcal{O}_{M}\right)^{\mu}
$$


This $\Gamma\left(U, \mathcal{O}_{M}\right)$-algebra has a basis of idempotents $f_{i}:=\delta_{i j}$ on $U_{j}$. Defining $e_{i} \in$ $\Gamma\left(U, \mathcal{T}_{M}\right)$ by $s\left(e_{i}\right)=f_{i}$, that is, $\bar{e}_{i} F \bmod J_{F}=f_{i}$, we get a local $\mathcal{O}_{M}$-basis of $\mathcal{T}_{M}$ satisfying $e_{i} \circ e_{j}=\delta_{i j} e_{j}$ and $e=\sum_{i} e_{i}$. Denote by $u^{i} \in \Gamma\left(U, \mathcal{O}_{M}\right)$ the restriction of $F$ to $U_{i}$ pushed down to $U$ that is, put

$$
u^{i}=F\left(\rho_{i}\right)
$$

in the notation of (2.1). Small subsets cover $M \backslash G$ so that the structure group of $\mathcal{T}_{M}$ is reduced to $S_{\mu}$. Summarizing, we have:

2.2.2. Proposition. The data $\left(\mathcal{T}_{M}^{f}, g, A\right)$ define on $M \backslash G$ the structure of semisimple pre-Frobenius manifold in the sense of [M], Ch. I, Def. 3.1 and 3.2. Moreover, we have $e_{i} u^{j}=\delta_{i j}$ so that $\left(u^{j}\right)$ form a local coordinate system (Dubrovin's canonical coordinates) and $\left[e_{i}, e_{j}\right]=0$ because $e_{i}=\partial / \partial u^{i}$.

Proof. Only the last statement might need some argumentation. We have $p^{*}\left(e_{i} u^{j}\right)=\bar{e}_{i} p^{*}\left(u^{j}\right)$ for any lift $\bar{e}_{i}$ of $e_{i}$. To calculate the right hand side we can restrict it to any local section of $p$ since it is constant along the fibers. We choose $\bar{e}_{i}$ tangent to $U_{j}$ and restrict the right hand side to $U_{j}$ where $p^{*}\left(u^{j}\right)$ coincides with $F$. The result is $\delta_{i j}$ by the definition of $e_{i}$.

For the future use, we can reformulate this as follows. Dualizing $s$ we get the isomorphism $s^{t}: \Omega_{M}^{1} \rightarrow \mathcal{H o m}_{\mathcal{O}_{M}}\left(p_{C *}\left(\mathcal{O}_{C}\right), \mathcal{O}_{M}\right)$. Then $s^{t}\left(d u^{i}\right): p_{C *}\left(\mathcal{O}_{C}\right) \rightarrow \mathcal{O}_{M}$ is the map which annihilates $j$-components for $j \neq i$ and coincides with the pushforward on the $i$-th component.

2.2.3. Theorem. The structure $\left(M, \mathcal{T}_{M}^{f}, g, A\right)$ associated to the Saito framework is Frobenius iff $d \epsilon=0$.

Proof. To check the Frobenius property on $M \backslash G$ we appeal to [M], Ch. I, Th. 3.3 (Dubrovin's criterium), both conditions of which, $\left[e_{i}, e_{j}\right]=0$ and $d \epsilon=0$ are satisfied. To pass from $M \backslash G$ to $M$ one can use a continuity argument, e. g. in the following form, again due to Dubrovin. Let $\nabla_{0}$ be the Levi-Civita connection of $g$, and $\nabla_{\lambda}$ the pencil of connections on $\mathcal{T}_{M}$ determined by its covariant derivatives $\nabla_{\lambda, X}(Y):=\nabla_{0, X}(Y)+X \circ Y$. Then $M$ if Frobenius iff $\nabla_{\lambda}$ is flat for some $\lambda \neq 0$, and so automatically for all $\lambda$. Clearly, this is the closed property.

We will now discuss when $e$ is flat.

On a small $U$, we can define functions $\eta_{j}$ by $\eta_{j}=i_{e_{j}}(\epsilon)=g\left(e_{j}, e_{j}\right)$. When $e_{j}=\partial / \partial u^{j}$, the closedness of $\epsilon=\sum_{i} \eta_{i} d u^{i}$ means that $\eta_{j}=e_{j} \eta$ for a local function $\eta$ well defined up to addition of a constant, or else $\epsilon=d \eta$. In the notations (2.1)

$$
\eta_{i}=\frac{1}{\operatorname{det}\left(\left(\partial^{2} F / \partial z_{a} \partial z_{b}\right)\left(\rho_{i}\right)\right)} .
$$

2.2.4. Theorem. Assume that the conditions of the Theorem 2.2.3. are satisfied.

The identity e is flat, iff for all $i, e \eta_{i}=0$, or equivalently, en $=g(e, e)=$ const. This holds automatically in the presence of an Euler field $E$ with $D \neq 2 d_{0}$ (see 1.2 above and 2.2.5 below).

This is Prop. 3.5 from [M], Ch. I.

One important remark about the identity is in order. Namely, in all examples I know of there exists a lift $\bar{e}$ of $e$ to $N$ such that $\bar{e} F=1$ identically, so that in the 
appropriate coordinate system we have $F=F_{0}+t_{0}$ where $F_{0}$ does not depend on $t_{0}$ which is lifted from $M$, and $\bar{e}=\partial / \partial t_{0}$.

It remains to clarify what Euler fields this structure can have.

2.2.5. Theorem. Assume that the conditions of the Theorem 2. 2.3. hold. Let $E$ be a vector field on a small subset $U$ in $M$.

a) We have $\operatorname{Lie}_{E}(\circ)=d_{0}$ o iff

$$
E=d_{0} \sum_{i}\left(u^{i}+c^{i}\right) e_{i}
$$

for some constants $c^{i}$, where $\left(d u^{i}\right)$ are 1-forms dual to $\left(e_{j}\right)$.

In particular, for non-zero $E$ we have $d_{0} \neq 0$ so that we may normalize $E$ by $d_{0}=$ 1. Furthermore, if the monodromy representation of the fundamental group of $M \backslash G$ on $H_{0}$ of the fibers of $C \rightarrow M$ has only one-dimensional trivial subrepresentation, the global vector field $E$ of this form with fixed $d_{0}$ is defined uniquely up to addition of a multiple of $e$.

b) For a field $E$ of the form (2.5) and a constant $D$, we have $\operatorname{Lie}_{E}(g)=D g$ iff

$$
E \eta=\left(D-d_{0}\right) \eta+\text { const. }
$$

In particular, if $e$ is flat, adding a multiple of $e$ does not change the validity of this property.

This follows from $[\mathrm{M}]$, Ch. I, Th. 3.6.

When $M$ comes from the Saito framework, we have a natural candidate for the global Euler field with $d_{0}=1$ suggested by our identification of local coordinates $u^{i}$. Namely, put on any small $U$

$$
E_{F}:=\sum_{i=1}^{\mu} F\left(\rho_{i}\right) e_{i}=\sum_{i=1}^{\mu} u^{i} e_{i} .
$$

Assume that it is in fact an Euler field and that we are in the conditions when it is defined uniquely up to a shift by a multiple of $e$. Assume furthermore that there exists a point 0 in $M$ to which $E_{F}$ extends and at which it vanishes ( 0 may lie in $G$, and in the theory of singularities it does so). Since $e$ cannot vanish, the choice of such 0 fixes $E_{F}$ completely.

2.2.6. Definition. Saito's framework $(p: N \rightarrow M, F, \omega)$ is called the strong Saito framework, if the structure $\left(M, \mathcal{T}_{M}^{f}, g, A\right)$ described above is Frobenius, with flat identity $e$ and Euler field $E_{F}$.

2.2.7. Remark. Since the definitions of the pre-Frobenius and Frobenius structures, and also of the identity and Euler fields, are local, we can lift all these structures from $M \backslash G$ to $C \backslash G_{C}$.

2.3. Unfolding singularities. K. Saito's theory (cf. [S1], [S2], [O] and the references therein) produces (a germ of) a strong Saito's framework starting with a germ of holomorphic function $f\left(z_{1}, \ldots, z_{n}\right)$ with isolated singularity at zero.

Namely, one can choose holomorphic germs $\phi_{0}=1, \phi_{1}, \ldots, \phi_{\mu-1}$ whose classes constitute a basis of the Milnor ring $\mathbf{C}\{\{z\}\} /\left(\partial f / \partial z_{a}\right)$ in such a way that $F:=$ $f+\sum t_{i} \phi_{i}$ is the miniversal unfolding of $f$. Then $N=N_{f}$, resp. $M=M_{f}$ is a neighborhood of zero in the $(z, t)-$, resp. $(t)$-space, and $F$ is defined above. 
The crucial piece of the structure is the choice of $\omega$ encoded in the Saito notion of a good primitive form. Generally its existence is established in an indirect way. For the singularities of $A D E$-type one can take $\omega=d z_{1} \wedge \cdots \wedge d z_{n}$. Generally, if $f$ is a quasi-homogeneous polynomial, most of the data constituting the Saito framework are algebraic varieties, rational maps and rational differential forms so that the whole setup has considerably more global character.

In order to help the reader to compare notation, we notice that Saito's $S$ is our $M$, and our function $\eta$ is denoted $\tau$ on p. 630 of [O]. Starting with the germ of $z^{n+1}$ at zero, one obtains in this way the following Frobenius manifold.

2.3.1. Example: manifolds $A_{n}$. Denote by $N$, resp. $M$, the affine space with coordinates $\left(z ; a_{1}, \ldots, a_{n}\right)$, resp. $\left.a_{1}, \ldots, a_{n}\right)$, and by $p$ the obvious projection. Put

$$
F=F\left(z ; a_{1}, \ldots, a_{n}\right)=z^{n+1}+a_{1} z^{n-1}+\cdots+a_{n} .
$$

Then $C$ iz given by the equation $F^{\prime}(z)=0$. We choose $\omega=d_{p} z$.

Making explicit the basic structures described above we get the following description of $A_{n}$. Consider the global covering of $M$ whose points consist of total orderings of the roots $\rho_{1}, \ldots, \rho_{n}$ of $F^{\prime}(z)$. On the semisimple part of it where $F^{\prime}(z)$ has no multiple roots and $u^{i}:=F\left(\rho_{i}\right)$ are local coordinates we have the flat metric

$$
g:=\sum_{i=1}^{n} \frac{\left(d u^{i}\right)^{2}}{F^{\prime \prime}\left(\rho_{i}\right)}
$$

with metric potential

$$
\eta=\frac{a_{1}}{n+1}=\frac{1}{n-1} \sum_{i<j} \rho_{i} \rho_{j}=-\frac{1}{2(n-1)} \sum \rho_{i}^{2} .
$$

Furthermore, $e, E$ and flat coordinates $x_{1}, \ldots, x_{n}$ can be calculated through $\left(a_{1}, \ldots, a_{n}\right)$ (which are generically local coordinates as well):

$$
\begin{gathered}
e=\partial / \partial a_{n}, \text { i. e., } e a_{n}=1, e a_{i}=0 \text { for } i<n . \\
E=\frac{1}{n+1} \sum_{i=1}^{n}(i+1) a_{i} \frac{\partial}{\partial a_{i}}
\end{gathered}
$$

$x_{i}$ are the first Laurent coefficients of the inversion of $w=\sqrt[n+1]{p(z)}=z+O(1 / z)$ near $z=\infty$ :

$$
z=w+\frac{x_{1}}{w}+\frac{x_{2}}{w^{2}}+\cdots+\frac{x_{n}}{w^{n}}+O\left(w^{-n-1}\right) .
$$

For the direct proof of these statements, see e.g. [M], Ch. I, 4.5.

The spectrum of $A_{n}$ is $D=\frac{n+3}{n+1}, d^{(i)}=\frac{i+1}{n+1}, 1 \leq i \leq n$, more precisely, $E x_{i}=\frac{i+1}{n+1} x_{i}$. Hence the $d$-spectrum is

$$
d^{(n)}=\frac{n-1}{n+1}, q_{i}^{(n)}=\frac{i}{n+1}, i=0, \ldots, n-1 .
$$

Now, $\Phi$ is analytic in $x_{a}$ and the spectrum of $-\operatorname{ad} E$ is strictly positive. Therefore $\Phi$ must be a polynomial in flat coordinates. One can check that its degree is precisely $n+2$. Hence for $n \geq 2$ it cannot be of qc-type, and by the method described in $\S 1$ we can define for $A_{n}$ only modified correlators with gravitational descendants. 
Comparing the spectrum of $A_{n}$ with that of the quantum cohomology of projective spaces, one can somewhat imaginatively say that $A_{n}$ represents "projective space of dimension $\frac{n-1}{n+1}$, with rank one cohomology in each dimension $\frac{i}{n+1}, 0 \leq i \leq n-1$ and with vanishing canonical class".

2.3.2. Example: Gepner's manifolds $V_{n, k}$. Let $n \geq 2, k \geq 1, h=n+k$. We will call Gepner's Frobenius manifold $V_{n, k}$ the manifold which is produced from the Saito's framework obtained by unfolding the polynomial

$$
f_{n, k}\left(z_{1}, \ldots, z_{n-1}\right):=\frac{1}{h} \sum_{i=1}^{n-1} y_{i}^{h}
$$

where $y$ and $z$ are related by

$$
\prod_{j=1}^{n-1}\left(1+y_{j} T\right)=1+\sum_{l=1}^{n-1} z_{l} T^{l} .
$$

In particular, if one assigns to $z_{l}$ weight $l, f_{n, k}$ becomes quasi-homogeneous of weight $h$. Its unfolding space is spanned by the classes of appropriate monomials, and a Zariski open dense subset $V_{n, k}$ of this space carries the structure of the Frobenius manifold as above. This subspace contains the point $m$ corresponding to the fusion potential

$$
g_{n, k}\left(z_{1}, \ldots, z_{n-1}\right):=f_{n+1, k-1}\left(z_{1}, \ldots, z_{n-1}, 1\right) .
$$

As D. Gepner ([Ge2]) proved, the tangent space $\mathcal{T}_{m} V_{n, k}$ with o-multiplication, that is, the Milnor algebra of $g_{n, k}$, is isomorphic to the Verlinde algebra (fusion ring) of the $s u(n)_{k}$ WZW model of the conformal field theory. Zuber in [Z] conjectured, and Varchenko and Gusein-Zade in [G-ZV] proved, that the lattice of the Verlinde algebra and the respective bilinear form can be interpreted in terms of vanishing cycles of $f_{n, k}$.

The total Frobenius manifold $V_{n, k}$ is thus a deformation of this fusion ring, in much the same way as quantum cohomology is the deformation of the usual cohomology ring.

2.4. Direct sum diagram. We will consider now three Saito's frameworks $(p: N \rightarrow M, F, \omega)$ and $\left(p_{i}: N_{i} \rightarrow M_{i}, F_{i}, \omega_{i}\right), i=1,2$. We will call the direct sum diagram any cartesian square

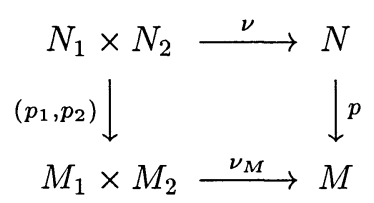

with the following properties:

(i) $\nu^{*}(F)=F_{1} \boxplus F_{2}$.

(ii) $\nu^{*}(\omega)=\omega_{1} \otimes \omega_{2}$.

Thus in a neighborhood of any point of $N$ lying over the image of $\nu_{M}$ there exist local coordinates $\left(z_{a}^{(1)}, z_{b}^{(2)} ; t_{e}\right)$ such that $t_{e}$ are lifted from $M$, and (i) can be written as

$$
F\left(z_{a}^{(1)}, z_{b}^{(2)} ; \nu_{M}^{*}\left(t_{e}\right)\right)=F_{1}\left(z_{a}^{(1)} ; t_{c}^{(1)}\right)+F_{2}\left(z_{b}^{(2)} ; t_{d}^{(2)}\right)
$$


and similarly (ii) can be written as

$$
\omega\left(z_{a}^{(1)}, z_{b}^{(2)} ; \nu_{M}^{*}\left(t_{e}\right)\right)=\omega_{1}\left(z_{a}^{(1)} ; t_{c}^{(1)}\right) \wedge \omega_{2}\left(z_{b}^{(2)} ; t_{d}^{(2)}\right) .
$$

2.4.1. Properties of the direct sum diagrams. Clearly, $\nu^{-1}(C)$ is defined by the equations $d_{p_{1}}\left(F_{1}\right) \boxplus d_{p_{2}}\left(F_{2}\right)=0$. Both summands then must vanish so that $\nu^{-1}(C)=C_{1} \times C_{2}$. Denote by $\nu_{C}: C_{1} \times C_{2} \rightarrow C$ the restriction of $\nu$. From (2.9) one then sees that

$$
\nu_{C}^{*}(\operatorname{Hess}(F))=\operatorname{Hess}\left(F_{1}\right) \otimes \operatorname{Hess}\left(F_{2}\right)
$$

and hence $\nu_{M}^{-1}(G)=G_{1} \times M_{2} \cup M_{1} \times G_{2}$. Let now $m=\nu_{M}\left(m_{1}, m_{2}\right), m_{i} \in M_{i}$. Choose small neighborhoods $m \in U$ in $M, m_{i} \in U_{i}$ in $M_{i}$ such that $\nu_{M}\left(U_{1} \times U_{2}\right) \subset U$. Number the connected components $U_{i}^{(1)}$ of $p_{C_{1}}^{-1}\left(U_{1}\right)$, resp. $U_{j}^{(2)}$ of $p_{C_{2}}^{-1}\left(U_{2}\right)$, by some indices $i$, resp. $j$, as in (2.1). Then the connected components of $p_{C}^{-1}(U)$ are naturally numbered by the ordered pairs $I=(i j)$ in such a way that

$$
\nu_{C}\left(U_{i}^{(1)} \times U_{j}^{(2)}\right) \subset U_{I}
$$

From now on we will assume that all the frameworks we are considering are strong ones. Then one can define $e_{I}, u^{I}, \eta_{I}$ etc as above, and from (2.3.), (2.4), (2.9)-(2.11) one immediately sees that

$$
u^{I}(m)=u_{1}^{i}\left(m_{1}\right)+u_{2}^{j}\left(m_{2}\right), \eta_{I}(m)=\eta_{i}^{(1)}\left(m_{1}\right) \eta_{j}^{(2)}\left(m_{2}\right)
$$

where in the right hand side we have the respective local functions on $M_{1}, M_{2}$.

The following slightly less evident restriction formula will be also needed in the next section.

2.4.2 Proposition. Let $I=(i j), K=(k l), \eta_{I K}=e_{I} \eta_{K}=e_{K} \eta_{I}$, and similarly $\eta_{i k}^{(1)}=e_{i} \eta_{k}^{(1)}$ etc. Then we have in the same notations as in (2.12):

$$
\eta_{I K}(m)=\delta_{j l} \eta_{i k}^{(1)}\left(m_{1}\right) \eta_{l}^{(2)}\left(m_{2}\right)+\delta_{i k} \eta_{k}^{(1)}\left(m_{1}\right) \eta_{j l}^{(2)}\left(m_{2}\right) .
$$

Proof. Calculate $\nu_{M}^{*}\left(d \eta_{I}\right)$ in two ways. On the one hand, we have

$$
\nu_{M}^{*}\left(d \eta_{I}\right)=\sum_{K} \nu_{M}^{*}\left(\eta_{I K}\right) \nu_{M}^{*}\left(d u^{K}\right) .
$$

As at the end of the proof of Prop. 2.2.2, we can identify $d u^{K}$ with a map from $p_{C *}\left(\mathcal{O}_{C}\right)$ to $\mathcal{O}_{M}$ vanishing on all components except for the $K$-th one where it is the canonical pushforward. After restriction to $M_{1} \times M_{2}$ it may therefore be non-vanishing only on $U_{k}^{(1)} \times U_{l}^{(2)}$ so that we can calculate $\nu_{M}^{*}\left(\eta_{I K}\right)$ by restricting $\nu_{M}^{*}\left(d \eta_{I}\right)$ to this product.

On the other hand, in view of (2.12),

$$
\begin{gathered}
\nu_{M}^{*}\left(d \eta_{I}\right)=d \nu_{M}^{*}\left(\eta_{I}\right)=d\left(\eta_{i}^{(1)} \otimes \eta_{j}^{(2)}\right)=d \eta_{i}^{(1)} \otimes \eta_{j}^{(2)}+\eta_{i}^{(1)} \otimes d \eta_{j}^{(2)}= \\
\sum_{r} \eta_{i r}^{(1)} d u_{1}^{r} \otimes \eta_{j}^{(2)}+\sum_{s} \eta_{i}^{(1)} \otimes \eta_{j s}^{(2)} d u_{2}^{s} .
\end{gathered}
$$

Only the $k$-th summand in the first sum restricted to $U_{k}^{(1)} \times U_{l}^{(2)}$ may be non-vanishing and considered as a map (c.f. above) it equals $\delta_{j l} \eta_{i k}^{(1)} \otimes \eta_{j}^{(2)}$ times the pushforward map. 
We have the similar expression for the $l$-th summand of the second sum. Comparison with (2.14) furnishes (2.13) because $\nu_{M}^{*}\left(d u^{K}\right)=d u_{1}^{k} \boxplus d u_{2}^{l}$.

2.5. Direct sums of singularities. In the theory of singularities, we can compare the miniversal unfolding spaces $M_{f}, M_{g}, M_{f+g}$ of the germs $f, g$ and $f+g$. It so happens that they fit into the direct sum diagram (2.8) (the only choice that remains is that of the volume form $\omega$ on the space of $f+g$ which is natural to take decomposable as in 2.4 above).

By iteration, we can consider arbitrary number of summands. In particular, the Frobenius manifold $A_{n_{1}, \ldots, n_{k}}$ which is obtained by unfolding the quasi-homogeneous singularity at zero $f(z):=z_{1}^{n_{1}}+\cdots+z_{k}^{n_{k}}$ is related to the summands $A_{n_{i}}$ in the way described above. We will show in the next section, that the formulas (2.12) and (2.13) imply a much neater description: $M_{f+g}=M_{f} \otimes M_{g}$, and in particular $A_{n_{1}, \ldots, n_{k}}$ is the tensor product of $A_{n_{i}}$ in the sense of [KMK] (in the context of formal Frobenius manifolds) and $[\mathrm{K}]$ (in the global context).

\section{Tensor products and their submanifolds.}

3.1. Tensor product of formal Frobenius manifolds. Let us first of all recall the general construction of the tensor product of formal Frobenius manifolds over a common coefficient ring $k$. Instead of $\left(M=\operatorname{Spf} k\left[\left[H^{t}\right]\right], g, \Phi\right)$ as in the Definition 1.4.1 we will be writing $(H, g, \Phi)$. We will not assume that our manifolds are of qc-type.

Let $\left(H^{(i)}, g^{(i)}, \Phi^{(i)}\right), i=1,2$, be two formal Frobenius manifolds. Then

$$
\left(H^{(1)}, g^{(1)}, \Phi^{(1)}\right) \otimes\left(H^{(2)}, g^{(2)}, \Phi^{(2)}\right)=\left(H^{(1)} \otimes H^{(2)}, g^{(1)} \otimes g^{(2)}, \Phi\right)
$$

where the terms of $\Phi$ of degree $n$ are defined in the following way: reconstruct the Cohomological Field Theories $I_{n}^{(i)}$ for the two factors as in 1.5, put $I_{n}:=I_{n}^{(1)} \cup I_{n}^{(2)}$ (cup product in $H^{*}\left(\bar{M}_{0, n}\right)$ ) and cap $I_{n}$ with the fundamental class of $\bar{M}_{0, n}$.

If the factors are endowed with flat identities and Euler fields, with $d_{0}^{(i)}=1$ for both of them, one can define in a canonical way the flat identity and the Euler field for the product $([\mathrm{K}])$. Moreover, the $d$-spectrum of the product is

$$
d=d^{(1)}+d^{(2)},\left\{q_{A}\right\}=\left\{q_{a}+q_{b}\right\}
$$

as a sum of families with multiplicities. Notice that if $\operatorname{ad} E$ is semisimple for both factors, it is semisimple for the product, and for $A=(a, b)$ one can identify $\left(\partial_{A}, q_{A}\right)$ with $\left(\partial_{a} \otimes \partial_{b}, q_{a}+q_{b}\right)$.

Since the tensor product potential is defined coefficientwise and involves multiplication in all cohomology algebras $H^{*}\left(\bar{M}_{0, n}\right), n \geq 3$, it is practically impossible to calculate it directly. The problem becomes much more manageable if we deal with (germs of) semisimple analytic manifolds.

The reason for this is that generally a germ of Frobenius manifold of a given dimension depends on functional parameters, even in the presence of a flat identity and Euler field. The semisimplicity condition puts sufficiently strong constraints in order to leave undetermined only a finite number of constants, and then it is reasonable to rxpect that the tensor product is calculable in terms of these constants. Below we review the relevant results following $[\mathrm{M}]$ and $[\mathrm{MM}]$.

3.2. Moduli space and tensor product of germs of semisimple Frobenius manifolds. Consider a pointed germ $M$ of analytic Frobenius manifold over $\mathbf{C}$, (or a 
formal manifold with zero as the base point), as usual, with flat $e$, Euler $E$ and $d_{0}=1$, and having pure even dimension. It will be called tame semisimple if the operator Eo has simple spectrum $\left(u_{0}^{1}, \ldots, u_{0}^{n}\right)$ on the tangent space to the base point. We have the following general facts already partly invoked in the specific situation of Saito's framework in 2.2 above:

a) In a neighborhood of the base point, eigenvalues $\left(u^{1}, \ldots, u^{n}\right)$ of $E$ o on $\mathcal{T}_{M}$ form a local coordinate system (Dubrovin's canonical coordinates), taking the values $\left(u_{0}^{1}, \ldots, u_{0}^{n}\right)$ at the base point. The potential $\Phi$ is an analytic function of these coordinates. If the initial manifold was only assumed to be formal, from tame semisimplicity it follows that it is in fact the completion of a pointed analytic germ.

b) Put $e_{i}=\partial / \partial u^{i}$. Then $e_{i} \circ e_{j}=\delta_{i j}$. In particular, $e=\sum_{i} e_{i}$. It follows that the - multiplication on the tangent spaces is semisimple.

c) We have $g\left(e_{i}, e_{j}\right)=0$ for $i \neq j$. Furthermore, there exists a function $\eta$ defined up to addition of a constant such that $g\left(e_{i}, e_{i}\right)=e_{i} \eta:=\eta_{i}$. Moreover, we have $e g=$ const, $E g=(D-1) \eta+$ const. Finally, $E=\sum_{i} u^{i} e_{i}$.

A very important feature of canonical coordinates is that a given tame semisimple germ can be uniquely extended to the Frobenius structure on the universal covering of the total $\left(u^{i}\right)$-space with deleted partial diagonals. This follows from the Painlevé property of the solutions of Schlesinger's equations: cf. [M], Ch. II, sec. 1-3. We will call this extension the maximal tame continuation of the initial germ. The qualification "tame" is essential. It may well happen that a further extension containing non-tame semisimple points or even points with non-semisimple multiplication on the tangent space is possible: e.g. points in $A_{n}$ where $F^{\prime}(z)$ has multiple roots have the latter property.

3.2.1. Definition. Special coordinates of a tame semisimple pointed germ of Frobenius manifold consist of the values at the base point of the following functions:

$$
\left(u^{i}, \eta_{j}, v_{i j}:=\frac{1}{2}\left(u^{j}-u^{i}\right) \frac{\eta_{i j}}{\eta_{j}}\right)
$$

Here $\eta_{i j}:=e_{i} e_{j} \eta$.

To avoid any misunderstanding, let us stress that the canonical coordinates are functions on a germ, whereas special coordinates are functions on the moduli space of germs.

For a description of the necessary and generically sufficient conditions for a system of mumbers to form special coordinates of a Frobenius germ, see [MM], 2.7, pp. 26-27, and 2.6, p. 23, where some inaccuracies of $[\mathrm{M}]$ are corrected. The following Theorem summarizes the properties of special coordinates that we will use.

3.2.2. Theorem. (i) Any tame semisimple pointed germ with labelled spectrum of Eo is uniquely (up to isomorphism) defined by its special coordinates.

(ii) Let $\left(u^{i}, \eta_{j}^{\prime}, v_{i j}^{\prime}\right)$ for $i \in S$ and $\left(u^{\prime \prime i}, \eta_{j}^{\prime \prime}, v_{i j}^{\prime \prime}\right)$ for $j \in T$ be special coordinates of two pointed germs. If the family $u^{i i}+u^{\prime \prime j}$ consists of pairwise distinct elements, then the tensor product of the two germs defined through their completions is again a tame semisimple pointed germ whose canonical coordinates are naturally labelled by the pairs $I \in S \times T$ and have the following form: for $I=(i, j), K=(k, l)$,

$$
u^{I}=u^{\prime i}+u^{\prime \prime j}, \eta_{I}=\eta_{i}^{\prime} \eta_{j}^{\prime \prime}, v_{I K}=\delta_{j l} v_{i k}^{\prime}+\delta_{i k} v_{j l}^{\prime \prime}
$$


(iii) Let another two germs be obtained from the initial ones by analytic continuation and subsequent shifts of base points. Then their tensor product can be obtained from the initial tensor product by analytic continuation and the appropriate shift of the base point. In this sense, the tensor product does not depend on the choice of base points.

The first statement is proved in $[\mathrm{M}]$ and $[\mathrm{MM}]$. The second and the third ones are due to R. Kaufmann ([K]). Actually, the third statement is proved in $[\mathrm{K}]$ in the considerably more general context: Kaufmann uses flat coordinates and does not assume semisimplicity or absence of odd coordinates. The fact that the tensor product of two convergent germs is again convergent is proved in his paper in preparation, without semisimplicity assumption as well.

Kaufmann remarks that in order to prove (3.2) it suffices to control the relevant potentials only to the fourth order in flat coordinates, and the necessary calculation can then be done directly.

We can now deduce from (3.2) the following corollary.

3.2.3. Theorem. Assume that we have the direct sum diagram of Saito's frameworks as in 2.4 above. Then the Frobenius manifold $M$ is (canonically isomorphic to) the tensor product of the Frobenius manifolds $M_{1} \otimes M_{2}$.

Proof. In the notation of (2.12) we may assume that $m_{1}, m_{2}$ and $m$ are tame semisimple, because tameness is the open property. Then (2.12) coincides with the first two formulas of (3.2). The third one follows directly from (2.13) and the definition of $v_{i j}$ in (3.1).

We will now prove that the integral part of the spectrum corresponds to a Frobenius submanifold.

3.3. Proposition. Assume that we have an analytic or formal Frobenius manifold $M$ with an Euler field $E, d_{0}=1, D \in \mathbf{Z}$, and flat identity. Let $-\operatorname{ad} E$ be semisimple on flat vector fields with spectrum $d_{a},\left(x_{a}\right)$ a flat coordinate system with

$$
E=\sum_{a: d_{a} \neq 0} d_{a} x^{a} \partial_{a}+\sum_{b: d_{b}=0} r_{b} \partial_{b} .
$$

and $e=\partial_{0}$. Define the submanifold $H M \subset M$ by the equations

$$
x_{c}=0 \text { for all } c \text { such that } d_{c} \notin \mathbf{Z} \text {. }
$$

Finally, assume that at least one of the following conditions is satisfied:

(i) $r_{b}=0$ for all $b$ with $\left|\Delta_{b}\right|=2$.

(ii) $M$ is of qc-type, and $\sum_{b: d_{b}=0} r_{b} \Delta_{b}$ takes only integral values on $B$.

Then HM with induced metric, o-multiplication, $E$ and $e$ is a Frobenius manifold.

REMARK. From the proof it will be clear that one can replace integers in this statement by any arithmetic progression containing 0 to which $D$ and $d_{0}$ belong.

Proof of the proposition 3.3. If $d_{c}$ is not integral, the functions $E x_{c}=d_{c} x_{c}, e x_{c}=$ 0 vanish on $H M$. Hence $E$ and $e$ are tangent to $H M$ and can be restricted to it. From the equation $\left(d_{a}+d_{b}-D\right) g_{a b}=0([\mathrm{M}]$, p. $32,(2.17))$ one sees that if $d_{a}, D \in \mathbf{Z}, d_{b} \notin$ $\mathbf{Z}$, we have $g_{a b}=0$. Therefore the restriction of $g$ to $H M$ is non-degenerate (it is obviously flat), and $x_{a}$ for $d_{a} \in \mathbf{Z}$ restrict to a flat coordinate system on $H M$. The 
o-product of two vector fields tangent to $H M$ at the points of $M$ does not contain the transverse components. In fact, we have $E \Phi_{a b}{ }^{c}=\left(d_{0}-d_{a}-d_{b}+d_{c}\right) \Phi_{a b}{ }^{c}([\mathrm{M}]$, p. 32, (2.18)). Hence if $d_{a}, d_{b} \in \mathbf{Z}, d_{c} \notin \mathbf{Z}$, then in the case (i) every monomial in the series $\Phi_{a b}^{c}$ must be an eigenvector of $E$ with non-integral eigenvalue, and therefore it must contain some $x_{e}$ with $d_{e} \notin \mathbf{Z}$ so that it vanishes on $H M$. In the case (ii) we apply the same reasoning separately to the generalized (involving exponentials) monomials contributing to the third derivatives of $\Psi$ in (1.9) and to the third derivatives of $c$.

The same reasoning shows that the induced multiplication of vector fields on $\mathcal{T}_{H M}$ is defined by the third derivatives of the induced potential.

3.4. Special coordinates of $A_{n}$. We return to the notation of 2.3.1.

3.4.1. Proposition. Consider the points of $A_{n}$ where $a_{1}, \ldots, a_{n-2}=0, a_{n-1}$, $a_{n}$ arbitrary. Choose a primitive root $\zeta$ of $\zeta^{n}=1$ and a root $b$ of $b^{n}=-\frac{a_{n-1}}{n+1}$. At these points we have:

$$
\begin{gathered}
u^{i}=a_{n}+\frac{n}{n+1} \zeta^{i} a_{n-1} b, \\
\eta_{i}=\frac{\zeta^{i}}{n(n+1) b^{n-1}}, \\
v_{j k}=\frac{1}{(n+1)\left(1-\zeta^{k-j}\right)} .
\end{gathered}
$$

REMARK. It is suggestive to compare these coordinates with those for the quantum cohomology of $\mathbf{P}^{n-1}$ ([M], p. 71) on the plane spanned by the identity (coordinate $x_{0}$ ) and the dual hyperplane section (coordinate $x_{1}$ ):

$$
\begin{gathered}
u^{i}=x_{0}+n \zeta^{i} e^{\frac{x_{1}}{n}} \\
\eta_{i}=\frac{\zeta^{i}}{n} e^{-x_{1} \frac{n-1}{n}} \\
v_{j k}=\frac{1}{1-\zeta^{k-j}} .
\end{gathered}
$$

Proof. At our subspace $F(z)=z^{n+1}+a_{n-1} z+a_{n}$. Hence

$$
F^{\prime}(z)=(n+1)\left(z^{n}+\frac{a_{n-1}}{n+1}\right)
$$

has roots $\rho_{i}=\zeta^{i} b$. But for $A_{n}$-manifolds we have universally $u^{i}=F\left(\rho_{i}\right), \eta_{i}=\frac{1}{F^{\prime \prime}\left(\rho_{i}\right)}$. This furnishes (3.3) and (3.4).

The proof of (3.5) is longer. We have to calculate the values of functions

$$
\frac{1}{2}\left(u^{k}-u^{j}\right) \frac{\eta_{j k}}{\eta_{k}}
$$

restricted to the plane of our base points. 
At a generic point of $A_{n}$, we can calculate $\eta_{j k}$ in the following three-step way:

$$
\eta_{j k}=\frac{\partial \eta_{j}}{\partial u^{k}}=\sum_{l, m=1}^{n} \frac{\partial \eta_{j}}{\partial \rho_{m}} \frac{\partial \rho_{m}}{\partial a_{l}} \frac{\partial a_{l}}{\partial u^{k}} .
$$

Since

$$
\eta_{j}=\frac{1}{F^{\prime \prime}(z)}=\frac{1}{(n+1) \prod_{i: i \neq j}\left(\rho_{i}-\rho_{j}\right)}
$$

we have

$$
\frac{\partial \eta_{j}}{\partial \rho_{m}}=-\frac{\eta_{j}}{\rho_{m}-\rho_{j}}
$$

if $m \neq j$, and

$$
\frac{\partial \eta_{j}}{\partial \rho_{j}}=\eta_{j} \sum_{i: i \neq j} \frac{1}{\rho_{i}-\rho_{j}}
$$

Moreover,

$$
\frac{\partial \rho_{m}}{\partial a_{l}}=-(n-l) \rho_{m}^{n-l-1} \eta_{m}
$$

This can be checked by derivating the identity $F^{\prime}\left(\rho_{m}\right)=0$.

Finally, according to [M], p. 47, (4.24), we have

$$
\sum_{l=1}^{n} \frac{\partial a_{l}}{\partial u^{k}} \rho_{i}^{n-l}=\delta_{i k}
$$

We will now restrict (3.8)-(3.11) to our plane.

Using (3.3) and (3.4), we get consecutively:

$$
\frac{\partial \eta_{j}}{\partial \rho_{m}}=\frac{1}{n a_{n-1}} \frac{1}{\zeta^{m-j}-1}
$$

if $m \neq j$, and

$$
\begin{gathered}
\frac{\partial \eta_{j}}{\partial \rho_{j}}=\frac{n-1}{2 n a_{n-1}}, \\
\frac{\partial \rho_{m}}{\partial a_{l}}=-\frac{n-l}{n(n+1)} b^{-l} \zeta^{-m l} .
\end{gathered}
$$

Solving (3.11) for partial derivatives, we also find

$$
\frac{\partial a_{l}}{\partial u^{k}}=\frac{1}{n} b^{l-n} \zeta^{k l} .
$$

It remains to substitute (3.12)-(3.14) into (2.7) to get after some calculation

$$
\eta_{j k}=\frac{2 \zeta^{k-j}}{\left(\zeta^{k-j}-1\right)^{2}} \frac{1}{n a_{n-1}^{2}} .
$$

Finally, substituting (3.3), (3.4) and (3.16) in (3.6), we obtain (3.5). 
3.5. Tensor products of $A_{n}$ 's. We want to describe $\left(n_{1}, \ldots, n_{N}\right)$ with nontrivial $H\left(A_{n_{1}} \otimes \cdots \otimes A_{n_{N}}\right)$. We can assume $n_{i} \geq 2$ because $A_{1}$ is identity with respect to the tensor multiplication. The first necessary condition, following from (1.20) is

$$
\mathbf{d}:=\sum_{i=1}^{N} \frac{n_{i}-1}{n_{i}+1} \in \mathbf{Z} .
$$

If it is satisfied, the full $d$-spectrum of the tensor product consists of certain rational points between 0 and $\mathbf{d}$. Multiplicity of 0 and $\mathbf{d}$ is one. Generally, the multiplicity of some $m \leq \mathbf{d}$ is

$$
\begin{gathered}
h^{2 m}\left(H\left(\otimes_{i} A_{n_{i}}\right)\right):=\text { the number of }\left(i_{1}, \ldots, i_{N}\right) \in \mathbf{Z}_{\geq 0}^{N} \text { satisfying } \\
\qquad \sum_{k=1}^{N} \frac{i_{k}}{n_{k}+1}=m, 0 \leq i_{k} \leq n_{k}-1 .
\end{gathered}
$$

The $d$-spectrum of $H\left(\otimes A_{n_{k}}\right)$ consists of the part of (3.18) for all integer $m$. Clearly, $(0.1)$ is a particular case of (3.18).

The flat part of $E$ in the total tensor product and in the $H$-part of it vanishes because it vanishes on all factors (cf. [K], Theorem 6.3).

Let us show that $\otimes_{k} A_{n_{k}}$ admits a tame semisimple base point which is the "sum" of the points the special coordinates of which we have calculated. Choose base points on all $A_{n_{k}}$ as in Prop. 3.4.1. For our purpose, we may even assume that $a_{n_{k}}=0$ on each $A_{n_{k}}$. Therefore, slightly changing notation of (3.3), we will assume that canonical coordinates of the base point of $A_{n_{k}}$ are of the form $u^{i}=\zeta_{k}^{i} c_{k}$, where $\zeta_{k}$ is the primitive root of unit of degree $n_{k}$ and $0 \leq i \leq n_{k}-1$. Then in view of the Theorem 3.2.2 for $I=\left(i_{1}, \ldots, i_{N}\right), J=\left(j_{1}, \ldots, j_{N}\right)$ we have a pair of canonical coordinates $u^{I}, u^{J}$ on the full tensor product whose values at the base point of this product are

$$
u^{I}=\sum_{k=1}^{N} \zeta_{k}^{i_{k}} c_{k}, u^{J}=\sum_{k=1}^{N} \zeta_{k}^{j_{k}} c_{k}
$$

One easily sees that with generic choice of $c_{k}$ these coordinates are distinct for all $I \neq J$.

3.6. Involutive pairs of Gepner's manifolds. In the notations of 2.3..2, consider a pair of Frobenius manifolds $V_{n+1, k}$ and $V_{k+1, n}$. They contain respectively points producing Verlinde's algebras of $s u(n+1)_{k}$ and $s u(k+1)_{n}$ which are isomorphic according to the reasoning of [G-ZV] (the level-rank duality). This reasoning runs as follows. Assume for concreteness that $n \geq k+1$. Consider the function

$$
\tilde{f}_{n+1, k}\left(z_{1}, \ldots, z_{n}\right):=f_{k+1, n}\left(z_{1}, \ldots, z_{k}\right)+\sum_{i=k+1}^{n} z_{i} z_{n+k-i+1} .
$$

Then $f_{n+1, k}$ and $\widetilde{f}_{n+1, k}$ are quasi-homogeneous polynomials of the same degree, depending on the same set of weighted variables, and having an isolated critical point at the origin. Hence they belong to a connected family of polynomials with the same property, and whatever structures can be derived from their lattices of vanishing cycles, they can be identified. On the other hand, $\widetilde{f}_{n+1, k}$ is obtained from $f_{k+1, n}\left(z_{1}, \ldots, z_{k}\right)$ by adding a sum of squares which again does not change the structure of vanishing cycles, except for that of the intersection form which changes in a controlled way. 
In fact, adding a sum of squares does not change the respective Frobenius manifolds: this agrees with the fact that $A_{1}$ is the tensor identity. Hence $V_{n+1, k}$, or at least its germ at the origin, is deformable to (the germ of) $V_{k+1, n}$.

Perhaps, these Frobenius manifolds, or at least their appropriate coverings, are themselves isomorphic. To check this, it would suffice to identify their special coordinates at an appropriate pair of tame semisimple points. The Gepner-Verlinde points $m \in V_{n, k}$ (cf. 2.3..2 above) are certainly not tame because the potential has only $n$, not $n+k$ different critical values at $m$.

\section{Maurer-Cartan and master equations.}

4.1. Maurer-Cartan equations. Fix a supercommutative $\mathbf{Q}$-algebra $k$. All our structures are $\mathbf{Z}_{2}$-graded, notation like $\widetilde{x}$ means the parity of a homogeneous element $x$. Let $g=g_{0} \oplus g_{1}$ be a Lie superalgebra over $k$, supplied with an odd differential $d$ satisfying $d[a, b]=[d a, b]+(-1)^{\tilde{a}}[a, d b]$.

Put $Z=Z(g, d):=\operatorname{Ker} d, B=B(g, d):=\operatorname{Im} d, H:=H(g, d)=Z / B$. Clearly, $Z$ is a Lie subalgebra, and $B$ its ideal, so that $H$ with induced bracket product is a Lie superalgebra.

The differential $d$ can be shifted.

For $\gamma \in g_{1}$, put $d_{\gamma}(a):=d a+[\gamma, a]$. Clearly, $d_{\gamma}[a, b]=\left[d_{\gamma} a, b\right]+(-1)^{\tilde{a}}\left[a, d_{\gamma} b\right]$.

4.1.1. Claim. a) We have $d_{\gamma}^{2}=0$ if

$$
d \gamma+\frac{1}{2}[\gamma, \gamma]=0
$$

b) Let $\gamma^{\prime}=\gamma+\epsilon \beta, \beta \in g, \epsilon$ an even or odd constant with $\epsilon^{2}=0$ such that $\epsilon \beta$ is odd. Assume that $\gamma$ satisfies (4.1). Then $\gamma^{\prime}$ satisfies (4.1) as well iff

$$
d_{\gamma}(\beta)=d \beta+[\gamma, \beta]=0
$$

This is straightforward.

If $K$ is another supercommutative $k$-algebra, we define $g_{K}=K \otimes_{k} g, d_{K}=1 \otimes d$. We will always work with $K$ flat over $k$ so that $Z_{K}:=\operatorname{Ker} d_{K}=K \otimes_{k} Z$, and similarly for $B$ and $H$. Claim 4.4.1 is of course applicable to $\left(g_{K}, d_{K}\right)$ as well.

We want to produce from this setting a non-linear version of the homology $H(g, d)$ or rather of the diagram $g \supset Z \rightarrow H$.

The most straightforward is the case when, say, $g$ is free of finite rank over $k$. We then replace $g$ by the linear superspace $\mathcal{G}:=\operatorname{Spec} k\left[\Pi g^{t}\right]$, where $\Pi$ is the parity inversion functor, $Z$ by the closed subspace $\mathcal{Z} \subset \mathcal{G}$ defined by the equations (4.1). In order to understand what should be the non-linear version of $B$, we interpret the Claim 4.1.1 (ii) as saying that $d_{\gamma}$-cycles form the Zariski tangent space to the point $\gamma$ of the Maurer-Cartan space (4.1). It then contains the subspace of $d_{\gamma}$-boundaries, and we can construct the distribution $\mathcal{B}$ generated by the boundaries. If the quotient space $\mathcal{H}=\mathcal{Z} / \mathcal{B}$ in some sense exists, it can be regarded as the non-linear cohomology of $(g, d)$.

In more down-to-earth terms, choose a (homogeneous) basis $\left\{\gamma_{i}\right\}$ of $g$ and a family of independent (super)commuting variables $t^{i}$ such that $\widetilde{t}^{i}=\widetilde{\gamma}_{i}+1$. Then $\Gamma:=\sum_{i} t^{i} \gamma_{i}$ is a generic odd element of $g$ (or rather of $k\left[t^{i}\right] \otimes g$ ), and the equation 
$d \Gamma+\frac{1}{2}[\Gamma, \Gamma]=0$ is equivalent to the system of equations

$$
\forall k: \quad \sum_{i}(-1)^{i} t^{i} D_{i}^{k}+\frac{1}{2} \sum_{i, j} t^{i} t^{j}(-1)^{i(j+1)} L_{i j}^{k}=0 .
$$

Here we define the structure constants by $d \gamma_{i}=\sum_{k} D_{i}^{k} \gamma_{k}$ and $\left[\gamma_{i}, \gamma_{j}\right]=\sum_{k} L_{i j}{ }^{k} \gamma_{k}$ and use the following shorthand for the signs: $(-1)^{i(j+1)}$ means $(-1)^{\tilde{\gamma}_{i}\left(\tilde{\gamma}_{j}+1\right)}$ etc.

These equations define the coordinate ring $R$ of the affine scheme which we called $\mathcal{Z}$. Obviously, $\mathcal{Z}$ represents the following functor on the category of supecommutative $k$-algebras $K$ :

$$
K \mapsto\left\{\text { solutions to }(4.1) \text { in }(K \otimes g)_{1}\right\} .
$$

Similarly, if we have any odd $d_{\Gamma}-$ cycle $\epsilon \beta=\sum_{a} \epsilon s^{a} \gamma_{a}$ with coefficients in $K \otimes R$, the statement $4.1 .1 \mathrm{~b}$ ) means that the map $X_{\beta}: t^{a} \rightarrow s^{a}$ descends to the derivation of $K \otimes R$ over $K$ that is, to a vector field on $\mathcal{G}_{K}$ of parity $\tilde{\epsilon}$. Of course, the adequate functorial language for derivations is that of the first order infinitesimal deformations of points, because generally the vector fields implied by $4.1 .1 \mathrm{~b}$ ) are defined only in the infinitesimal neighborhood of $\gamma$.

We will stop now discussing the case of finite rank $g$ because in most interesting examples this does not hold, and only $H(g, d)$ is of finite rank.

So we step back and try to produce a formal section of $\mathcal{Z}$ passing through $\gamma=0$ and transversal to the distribution $\mathcal{B}$. We want it to be of the same size as $H$, or rather $\Pi H$, and we will assume henceforth that $H$ is free of finite rank. From now on in this section, we denote $K:=k\left[\left[\Pi H^{t}\right]\right]=k\left[\left[x_{i}\right]\right]$ where $x_{i}$ are coordinate functions on $\Pi H$ dual to a basis of $\Pi H$. Any element $\Gamma \in g_{K}$ can be uniquely written as $\sum_{n \geq 0} \Gamma_{n}$ where $\Gamma_{n}$ is homogeneous of degree $n$ in $x_{i}$. Such an element can be naturally called a formal section of $\mathcal{Z}$, or a generic (formal) solution to (4.1), if it has the following properties:

a) $\Gamma \in\left(g_{K}\right)_{1}, \Gamma_{0}=0, \Gamma_{1}=\sum_{i} x_{i} c_{i}$ where $d c_{i}=0$ and classes of $c_{i}$ form a basis of $H$ odd dual to $\left\{x_{i}\right\}$.

b) $d_{K} \Gamma+\frac{1}{2}[\Gamma, \Gamma]=0$.

The necessary condition for the existence of $\Gamma$ is the identical vanishing of the Lie bracket induced on $H(g, d)$. In fact, the equation $d \Gamma+\frac{1}{2}[\Gamma, \Gamma]=0$ implies (assuming a) above) $d \Gamma_{2}+\frac{1}{2}\left[\Gamma_{1}, \Gamma_{1}\right]=0$. Hence $\left[c_{i}, c_{j}\right] \in B$. However, generally it is not sufficient. In fact, the next equation reads $d \Gamma_{3}+\left[\Gamma_{1}, \Gamma_{2}\right]=0$, but since $\Gamma_{2}$ may be non-closed, we cannot conclude that $\left[\Gamma_{1}, \Gamma_{2}\right]$ is a boundary. The manageable sufficient condition is stronger: $(g, d)$ must be quasi-isomorphic to the differential Lie algebra $H(g, d)$ with zero bracket and zero differential. For a considerably more general treatment see $[\mathrm{GoM}]$. Our direct and elementary approach is self-contained and produces slightly more detailed information in the cases essential for the theory of Frobenius manifolds.

4.2. Theorem. (i) Assume that there exists a surjective morphism of differential Lie superalgebras $\phi:(g,[], d,) \rightarrow(H, 0,0)$ inducing isomorphism on the homology. Then there exists a generic formal solution $\Gamma$ to (4.1).

Moreover, $\Gamma$ can be chosen in such a way that for any $n \geq 2, \Gamma_{n} \in K \otimes \operatorname{Ker} \phi$. In other words, $(\mathrm{id} \otimes \phi)(\Gamma)=\sum_{i} x_{i}\left[c_{i}\right]$. Such a solution will be called normalized. 
(ii) If (i) is satisfied, then for any generic solution $\Gamma$, non necessarily normalized, the map $\phi_{K}=\mathrm{id} \otimes \phi: g_{K} \rightarrow H_{K}$ is the surjective morphism of differential Lie superalgebras $\left(g_{K},[,]_{K}, d_{K, \Gamma}\right) \rightarrow\left(H_{K}, 0,0\right)$ inducing isomorphism on the homology.

Proof. (i) Let $n \geq 1$. Assuming that $\Gamma_{i}$ for $i \leq n$ are already constructed, and writing $d$ instead of $d_{K}$ we must find $\Gamma_{n+1}$ from the equation

$$
d \Gamma_{n+1}=-\frac{1}{2} \sum_{i, j: i+j=n+1}\left[\Gamma_{i}, \Gamma_{j}\right] .
$$

First of all we check that the right hand side of (4.5) is closed in $g_{K}$. In fact, since the components $\Gamma_{1}, \ldots, \Gamma_{n}$ satisfy the similar equations by the inductive assumption, the differential of the rhs equals

$$
\frac{1}{2} \sum_{i+j+k=n+1}\left[\left[\Gamma_{i}, \Gamma_{j}\right], \Gamma_{k}\right] .
$$

This expression vanishes because the Jacobi identity for odd elements reads

$$
\left[\left[\Gamma_{i}, \Gamma_{j}\right], \Gamma_{k}\right]+\left[\left[\Gamma_{k}, \Gamma_{i}\right], \Gamma_{j}\right]+\left[\left[\Gamma_{j}, \Gamma_{k}\right], \Gamma_{i}\right]=0
$$

Hence the coefficients of the rhs of (4.5) (as polynomials in $x_{i}$ ) belong to $Z \cap[g, g]$. But $[g, g] \in \operatorname{Ker} \phi$ and $Z \cap \operatorname{Ker} \phi=B$ because $\phi$ is a quasi-isomorphism. Thus we can solve (4.5).

We can add to any solution elements of $Z_{K}$ of degree $n+1$. But $Z+\operatorname{Ker} \phi=g$ because $\phi$ induces surjection on homology. Hence we can normalize $\Gamma_{n+1}$ by the requirement $\Gamma_{n+1} \in K \otimes \operatorname{Ker} \phi$.

(ii) Now fix $\Gamma$ satisfying (4.5) for all $n$. We will write $d_{\Gamma}$ instead of $d_{K, \Gamma}$ and put $Z_{\Gamma}:=\operatorname{Ker} d_{\Gamma} \subset g_{K}, B_{\Gamma}=d_{\Gamma}\left(g_{K}\right)$. We have $B \subset \operatorname{Ker} \phi_{K}$ and $\left[g_{K}, g_{K}\right] \subset \operatorname{Ker} \phi_{K}$, hence $B_{\Gamma} \subset \operatorname{Ker} \phi_{K}$. Therefore, $\phi_{K}$ is compatible with zero bracket and zero differential on $H_{K}$. The natural inclusion $Z_{\Gamma}+\operatorname{Ker} \phi_{K} \rightarrow g_{K}$ becomes surjection after the reduction modulo the ideal $\left(x_{i}\right)$ of $K$, because $\phi$ is surjective. Hence this inclusion is surjective, and $\phi_{K}$ is surjective as well. It remains to show that $\phi_{K}$ induces injection on homology, that is,

$$
Z_{\Gamma} \cap \operatorname{Ker} \phi_{K} \subset B_{\Gamma}
$$

Let $c=\sum_{n \geq 0} c_{n} \in Z_{\Gamma}$. This means that $d c_{0}=0$ and in general

$$
d c_{n}=-\sum_{i+j=n}\left[\Gamma_{i}, c_{j}\right]
$$

(we keep writing $d$ for $d_{K}$ ). Assuming that $\phi_{K}(c)=0$ we want to deduce the existence of homogeneous elements $a_{n}$ of degree $n$ in $g_{K}$ such that

$$
c_{n+1}=d a_{n+1}+\sum_{i+j=n+1}\left[\Gamma_{i}, a_{j}\right] .
$$

We have $d c_{0}=0$ and $\phi\left(c_{0}\right)=0$, hence $c_{0}$ is a boundary because $\phi$ is the quasiisomorphism. Assuming that $a_{0}, \ldots, a_{n}$ are found, we will establish the existence of $a_{n+1}$ satisfying (4.8), if we manage to prove that $c_{n+1}-\sum_{i+j=n+1}\left[\Gamma_{i}, a_{j}\right]$ is $d$-closed. In fact, this element also belongs to $\operatorname{Ker} \phi_{K}$ and so must be a boundary. 
The differential of this element is

$$
d c_{n+1}+\sum_{i+j=n+1}\left[\Gamma_{i}, d a_{j}\right]-\sum_{i+j=n+1}\left[d \Gamma_{i}, a_{j}\right]
$$

Replace in the first sum $d a_{j}$ by $c_{j}-\sum_{k+l=j}\left[\Gamma_{k}, a_{l}\right]$ for $j \leq n$ (this holds by induction). Replace in the second sum $d \Gamma_{i}$ by the sum of commutators from (4.5). The terms containing $c_{j}$ will cancel thanks to (4.7). The remaining terms can be written as

$$
-\sum_{i+j+k=n+1}\left[\Gamma_{i},\left[\Gamma_{j}, a_{k}\right]\right]+\frac{1}{2} \sum_{i+j+k=n+1}\left[\left[\Gamma_{i}, \Gamma_{j}\right], a_{k}\right] \text {. }
$$

This expression vanishes because of Jacobi identity.

4.2.1. Corollary. Define the map $\psi=\psi_{\Gamma}: H_{K} \rightarrow g_{K}$ as the $K$-linear extension of

$$
H \rightarrow g_{K}: X \mapsto \bar{X} \Gamma
$$

where $\bar{X}$ acts on $K \otimes g$ as the right $g$-linear extension of the derivation on $K$ acting as $(\Pi X, *)$ on $\Pi H^{t}$.

Then $\psi$ is a section of $\phi_{K}$ if $\Gamma$ is normalized.

Proof. First of all, we have $\bar{X}\left(d \Gamma+\frac{1}{2}[\Gamma, \Gamma]\right)=0$ from which it follows that $d_{\Gamma}(\bar{X} \Gamma)=0$, that is, $\bar{X} \Gamma$ is a $d_{\Gamma}$-cycle. Its image in $H_{K}$ is $\left(\bar{X} \Gamma_{1}+\sum_{n>2} \bar{X} \Gamma_{n}\right) \bmod B_{\Gamma}$. The first term is clearly $X$. The remaining ones are in $K \otimes \operatorname{Ker} \phi_{K}$, if $\Gamma$ is normalized.

4.3. Odd Lie (super)algebras. As in 4.1, let now $g=g_{0} \oplus g_{1}$ be a $k$-module endowed with a bilinear operation odd bracket $(a, b) \mapsto[a \bullet b]$ which satisfies the following conditions:

a) parity of $[a \bullet b]$ equals $\tilde{a}+\widetilde{b}+1$,

b) odd anticommutativity:

$$
[a \bullet b]=-(-1)^{(\tilde{a}+1)(\tilde{b}+1)}[b \bullet a],
$$

c) odd Jacobi identity:

$$
[a \bullet[b \bullet c]]=[[a \bullet b] \bullet c]+(-1)^{(\widetilde{a}+1)(\tilde{b}+1)}[b \bullet[a \bullet c]] .
$$

Such a structure will be called an odd Lie (super)algebra. We consider such algebras endowed with an odd differential satisfying

$$
d[a \bullet b]=[d a \bullet b]+(-1)^{\tilde{a}+1}[a \bullet d b] .
$$

Physicists sometimes denote such multiplication $\{$,$\} (see e.g. [LZ]). Our choice of$ notation allows one to use consistently the standard sign mnemonics of superalgebra, if $\bullet$ counts as an element of parity one.

If $(g, d)$ is the usual differential Lie superalgebra, the parity change functor $g \mapsto$ $\Pi g$ turns the usual bracket product [,] into the odd bracket product, and defines an equivalence of the two categories (the differential changes sign). It seems therefore that there is not much point in considering odd brackets. However, in the context of GBV-algebras they come together with usual supercommutative multiplication, and parity change then turns this multiplication into odd one (see the next section). This is, of course, a particular case of the general operadic formalism over the category of superspaces, where any operation can be inherently even or odd. 
In the next section we choose to work with even multiplication and odd bracket product. But we will use the results of this section, with appropriately modified parities and signs, for odd Lie superalgebras. In particular, the odd Maurer-Cartan equation in the physical literature is called the master equation:

$$
d \Gamma+\frac{1}{2}[\Gamma \bullet \Gamma]=0 .
$$

The Theorem 4.2 provides conditions of its solvability in $k\left[\left[H^{t}\right]\right] \otimes g$ rather than $k\left[\left[\Pi H^{t}\right]\right] \otimes g$. Notice also that $\Gamma$ in (4.13) must be even.

\section{Gerstenhaber-Batalin-Vilkovyski algebras.}

5.1. Gerstenhaber-Batalin-Vilkovyski algebras. Let $\mathcal{A}$ be a supercommutative algebra with identity over another supercommutative algebra $k$ (constants). Consider an odd $k$-linear operator $\Delta: \mathcal{A} \rightarrow \mathcal{A}, \Delta(1)=0$, with the following property:

$$
\forall a \in \mathcal{A}, \quad \partial_{a}:=(-1)^{\tilde{a}}\left(\left[\Delta, l_{a}\right]-l_{\Delta a}\right)
$$

is the derivation of parity $\tilde{a}+1$ over $k$.

Here $l_{a}$ denotes the operator of left multiplication by $a$, and brackets denote the supercommutator. Explicitly,

$$
\partial_{a} b=(-1)^{\tilde{a}} \Delta(a b)-(-1)^{\tilde{a}}(\Delta a) b-a \Delta b .
$$

The sign ensures the identity $\partial_{c a}=c \partial_{a}$ for any constant $c$. By definition of derivation,

$$
\left[\partial_{a}, l_{b}\right]=l_{\partial_{a} b} .
$$

The pair $(\mathcal{A}, \Delta)$ is called a $G B V$-algebra if, in addition, $\Delta^{2}=0$. There is an obvious operation of scalar extension.

5.1.1. Lemma. In any $G B V$-algebra we have

$$
\begin{aligned}
& {\left[\Delta, \partial_{a}\right]=\partial_{\Delta a},} \\
& {\left[\partial_{a}, \partial_{b}\right]=\partial_{\partial_{a} b}}
\end{aligned}
$$

Proof. From (5.1) we have

$$
\left[\Delta, \partial_{a}\right]=(-1)^{\tilde{a}}\left(\left[\Delta,\left[\Delta, l_{a}\right]\right]-\left[\Delta, l_{\Delta a}\right]\right) .
$$

From the Jacobi identity for operators and $[\Delta, \Delta]=0$ we find $\left[\Delta,\left[\Delta, l_{a}\right]\right]=0$ because

$$
\left.\left[\Delta,\left[\Delta, l_{a}\right]\right]=\left[[\Delta, \Delta], l_{a}\right]-\left[\Delta,\left[\Delta, l_{a}\right]\right]\right)
$$

From (5.1) with $\Delta a$ replacing $a$ we have $\left[\Delta, l_{\Delta a}\right]=(-1)^{\tilde{a}+1} \partial_{\Delta a}$. Hence

$$
\left[\Delta, \partial_{a}\right]=(-1)^{\tilde{a}+1}\left[\Delta, l_{\Delta a}\right]=\partial_{\Delta a} .
$$

To prove (5.4), we notice that since $\left[\partial_{a}, \partial_{b}\right]$ must be a derivation, in the intermediate calculations we are allowed not to register all the summands which are left multiplications: they will cancel anyway. So we have, denoting such summands by dots and using consecutively (5.1), (5.2), Jacobi and (5.3), and again (5.1) with $\partial_{a} b$ replacing $a:$

$$
\left[\partial_{a}, \partial_{b}\right]=(-1)^{\tilde{b}}\left[\partial_{a},\left[\Delta, l_{b}\right]-l_{\Delta b}\right]=(-1)^{\tilde{b}}\left[\partial_{a},\left[\Delta, l_{b}\right]\right]+\ldots=
$$




$$
(-1)^{\tilde{b}+\widetilde{a}+1}\left[\Delta,\left[\partial_{a}, l_{b}\right]\right]+\ldots=(-1)^{\tilde{b}+\widetilde{a}+1}\left[\Delta, l_{\partial_{a} b}\right]+\ldots=\partial_{\partial_{a} b}
$$

Define now the odd bracket operation on $\mathcal{A}$ by the formula

$$
[a \bullet b]:=\partial_{a} b \text {. }
$$

5.1.2. Proposition. The pair of bilinear operations (multiplication and odd bracket) defines on $\mathcal{A}$ the structure of the odd Poisson algebra in the following sense:

(i) The odd bracket satisfies the odd anticommutativity, the odd Jacobi and the odd Poisson identities:

$[a \bullet b]=-(-1)^{(\widetilde{a}+1)(\tilde{b}+1)}[b \bullet a], \quad[a \bullet[b \bullet c]]=[[a \bullet b] \bullet c]+(-1)^{(\widetilde{a}+1)(\widetilde{b}+1)}[b \bullet[a \bullet c]]$,

$$
[a \bullet b c]=[a \bullet b] c+(-1)^{\widetilde{b}(\widetilde{a}+1)} b[a \bullet c] .
$$

(ii) $\Delta$ is the derivation with respect to the odd brackets so that $(\mathcal{A}, \bullet, \Delta)$ is the differential odd Lie algebra.

Proof. The anticommutativity can be checked directly. The Jacobi identity follows from $(5.4)$ written as $\left[\partial_{a}, \partial_{b}\right]=\partial_{[a \bullet b]}$. The Poisson identity means that $\partial_{a}$ is a derivation. The last statement follows from (5.1).

Notice that with respect to the usual multiplication $\Delta$ is the differential operator of order $\leq 2$ and not necessarily derivation.

5.2. Additional differential. Assume now that we have an additional $k$-linear odd map $\delta: \mathcal{A} \rightarrow \mathcal{A}$ which is the derivation with respect to the multiplicative structure of $\mathcal{A}$ satisfying

$$
\delta^{2}=[\delta, \Delta]=\delta \Delta+\Delta \delta=0
$$

We will say that $(\mathcal{A}, \Delta, \delta)$ is a differential $G B V$-algebra $(d G B V)$.

5.2.1. Lemma. We have

$$
\left[\delta, \partial_{a}\right]=\partial_{\delta a} .
$$

Therefore $\delta$ is the derivation with respect to the odd bracket as well.

Proof. Since $\left[\delta, \partial_{a}\right]$ is a derivation of $\mathcal{A}$, we can calculate omitting the multiplication operators as above:

$$
\begin{gathered}
{\left[\delta, \partial_{a}\right]=(-1)^{\tilde{a}}\left[\delta,\left[\Delta, l_{a}\right]\right]+\ldots=(-1)^{\tilde{a}}\left(\left[[\delta, \Delta], l_{a}\right]-\left[\Delta,\left[\delta, l_{a}\right]\right]\right)+\ldots=} \\
-(-1)^{\tilde{a}}\left[\Delta, l_{\delta a}\right]+\ldots=\partial_{\delta a} .
\end{gathered}
$$

Furthermore,

$$
\begin{gathered}
\delta[a \bullet b]=\delta \partial_{a} b=\left[\delta, \partial_{a}\right] b+(-1)^{\tilde{a}+1} \partial_{a} \delta b= \\
=\partial_{\delta a} b+(-1)^{\tilde{a}+1} \partial_{a} \delta b=[\delta a \bullet b]+(-1)^{\tilde{a}+1}[a \bullet \delta b] .
\end{gathered}
$$

5.2.2. Shifted differential. Let $\delta$ be a differential satisfying (5.7). For an even $a \in \mathcal{A}$ put

$$
\delta_{a}:=\delta+\partial_{a}, \delta_{a}(b)=\delta b+[a \bullet b] .
$$

Then we have $\delta_{a}^{2}=0$ if the odd Maurer-Cartan equation is satisfied:

$$
\delta a+\frac{1}{2}[a \cdot a]=0 .
$$


Furthermore,

$$
\left[\delta_{a}, \Delta\right]=0 \quad \text { if } \quad \Delta a=0 .
$$

Therefore, from $(5.11),(5.12)$ it follows that $\left(\mathcal{A}, \Delta, \delta_{a}\right)$ is a differential GBV-algebra (dGBV). In particular,

$$
\left[\delta_{a}, \partial_{b}\right]=\partial_{\delta_{a} b}
$$

We can in the same way shift $\Delta$. The essential difference is that, as $\Delta$ itself, the shifted differential generally will not be the derivation with respect to the associative multiplication.

5.3. Homology of $(\mathcal{A}, \delta)$. Since $\delta$ is the derivation with respect to both multiplications in $\mathcal{A}$ (associative one and the bracket), Ker $\delta$ is the subalgebra with respect to both of them, and $\operatorname{Im} \delta$ is the ideal in this subalgebra with respect to both structures. Therefore the homology group $H(\mathcal{A}, \delta)$ inherits both multiplications, satisfying the identities (5.6) and (5.7).

This reasoning holds for $H\left(\mathcal{A}, \delta_{a}\right)$ as well, if $a$ satisfies the Maurer-Cartan equation (5.11).

5.4. Homology of $(\mathcal{A}, \Delta)$. The same reasoning furnishes only the structure of odd Lie algebra on $H(\mathcal{A}, \Delta)$, because $\Delta$ is not a derivation with respect to the associative multiplication. However, if $\delta$ and $\Delta$ satisfy conditions (A) and (B) below, we will have the natural isomorphism $H(\mathcal{A}, \Delta)=H(\mathcal{A}, \delta)$.

The Lemma below is well known, see e.g. [GoM].

5.4.1. Lemma. Let $\mathcal{A}$ be an additive group supplied with two endomorphisms $\delta$ and $\Delta$ satisfying $\delta^{2}=\Delta^{2}=0$ and $\delta \Delta=\alpha \Delta \delta$ where $\alpha$ is an automorphism of $\mathcal{A}$ such that $\alpha(\operatorname{Im} \Delta \delta)=\operatorname{Im} \Delta \delta$. Then clearly, $\operatorname{Im} \delta \Delta=\operatorname{Im} \Delta \delta \subset \operatorname{Im} \delta \cap \operatorname{Ker} \Delta$ and similarly with $\delta$ and $\Delta$ permuted.

The following statements are equivalent:

(i) The inclusions of the differential subgroups $i:(\operatorname{Ker} \Delta, \delta) \subset(\mathcal{A}, \delta)$ and $j$ : $(\operatorname{Ker} \delta, \Delta) \subset(\mathcal{A}, \Delta)$ are quasi-isomorphisms (that is, induce isomorphisms of homology).

(ii) We have actually equalities:

$$
\begin{aligned}
& \operatorname{Im} \delta \Delta=\operatorname{Im} \Delta \delta=\operatorname{Im} \delta \cap \operatorname{Ker} \Delta, \\
& \operatorname{Im} \delta \Delta=\operatorname{Im} \Delta \delta=\operatorname{Im} \Delta \cap \operatorname{Ker} \delta .
\end{aligned}
$$

Assume that these conditions are satisfied. Then the both homology groups in (i) are naturally isomorphic to

$(\operatorname{Ker} \Delta \cap \operatorname{Ker} \delta) / \operatorname{Im} \delta \Delta$.

Moreover, the natural map $\operatorname{Ker} \Delta \rightarrow H(\mathcal{A}, \Delta)$ induces the surjection of the differential groups $(\operatorname{Ker} \Delta, \delta) \rightarrow(H(\mathcal{A}, \Delta), 0)$ which is a quasi-isomorphism, and similarly with $\delta$ and $\Delta$ interchanged. Hence the both differential groups $(\mathcal{A}, \Delta)$ and $(\mathcal{A}, \delta)$ are formal.

Proof. We have:

$$
H(i) \text { is injective } \Longleftrightarrow \operatorname{Ker} \Delta \cap \operatorname{Im} \delta=\delta(\operatorname{Ker} \Delta) .
$$




$$
H(i) \text { is surjective } \Longleftrightarrow \operatorname{Ker} \delta \subset \operatorname{Ker} \Delta+\operatorname{Im} \delta \Rightarrow \Delta(\operatorname{Ker} \delta)=\operatorname{Im} \delta \Delta .
$$

Here and below all kernel and images are taken in $\mathcal{A}$. In the right hand side of (5.14), the inclusion $\supset$ is evident, and the injectivity of $H(i)$ supplies the reverse inclusion. The last arrow in (5.15) is obtained by applying $\Delta$ to the previous inclusion: this gives $\Delta(\operatorname{Ker} \delta) \subset \Delta(\operatorname{Im} \delta)=\operatorname{Im} \delta \Delta$ whereas the reverse inclusion is obvious.

Interchanging $\delta$ and $\Delta$ we find

$$
H(j) \text { is injective } \Longleftrightarrow \operatorname{Ker} \delta \cap \operatorname{Im} \Delta=\Delta(\operatorname{Ker} \delta) .
$$

Taken together, (5.14) and (5.17) prove (A), and (5.15) and (5.16) prove (B), so that we have established the implication (i) $\Rightarrow$ (ii).

Conversely, assume that (A) and (B) hold.

Then $H(i)$ induces surjection on the homology, because if $\delta a=0$, we have $\Delta a \epsilon$ $\operatorname{Ker} \delta \cap \operatorname{Im} \Delta$ so that by (B), $\Delta a=\Delta \delta b$, and then $a-\delta b \in \operatorname{Ker} \Delta$ represents the same homology class as $a$.

Moreover, $H(i)$ induces injection on the homology, because if $a \in \operatorname{Ker} \Delta, a=\delta b$ for some $b \in \mathcal{A}$, then $a \in \operatorname{Ker} \Delta \cap \operatorname{Im} \delta$ so that by (A), $a=\delta c$ for some $c \in \operatorname{Im} \Delta \subset$ $\operatorname{Ker} \Delta$.

By symmetry, the same holds for $H(j)$.

The cycle subgroup for both differential groups $(\operatorname{Ker} \delta, \Delta)$ and $(\operatorname{Ker} \Delta, \delta)$ is $(\operatorname{Ker} \delta$ $\cap \operatorname{Ker} \Delta$ ), and if (i) and (ii) hold, the boundaries can be identified with $\operatorname{Im} \delta \Delta$, cf. (5.15) and (5.17). It remains to deduce formality, say, from (A) and (B).

The natural map Ker $\Delta \rightarrow H(\mathcal{A}, \Delta)$ is compatible with differentials, because if $a \in \operatorname{Ker} \Delta$, then $\delta a \in \operatorname{Im} \delta \cap \operatorname{Ker} \Delta$ so that by (A), $\delta a=\Delta \delta b$ for some $b$, and hence the map is compatible with the zero differential on $H(\mathcal{A}, \Delta)$.

This map is surjective on the homology. In fact, consider the class of $a, \Delta a=0$ in $H(\mathcal{A}, \Delta)$. Then $\delta a \in \operatorname{Im} \delta \cap \operatorname{Ker} \Delta$ so that in view of $(\mathrm{A}), \delta a=\delta \Delta b$, and the $\delta$-cycle $a-\Delta b$ represents the same class as $a$.

Finally, the map is injective on the homology. In fact, if $a \in \operatorname{Im} \Delta$ and $\delta a=0$, then in view of $(\mathrm{B}), a \in \operatorname{Im} \delta \Delta \subset \delta(\operatorname{Ker} \Delta)$.

Thus we established the two-step quasi-isomorphism of $(\mathcal{A}, \delta)$ with $(H(\mathcal{A}, \Delta), 0)$ and by symmetry of $(\mathcal{A}, \Delta)$ with $(H(\mathcal{A}, \delta), 0)$. But the first two groups are also naturally quasi-isomorphic. So they are formal.

5.4.2. Remarks. In the context of $d G B V-a l g e b r a s$, we will apply this identification to $\left(\mathcal{A}, \Delta, \delta_{a}\right)$ with variable or formal generic $a$. Then we will be able to interpret the "constant" space $H=H(\mathcal{A}, \Delta)$ as the flat structure on the family of algebras $H\left(\mathcal{A}, \delta_{a}\right)$ parametrized by the points of the generic formal section of the Maurer-Cartan manifold. The important technical problem will be then deriving the conditions (A) and (B) for the variable $a$.

Notice that taken together, (A) and (B) are equivalent to

$$
\operatorname{Im} \delta \Delta=\operatorname{Im} \Delta \delta=(\operatorname{Ker} \delta \cap \operatorname{Ker} \Delta) \cap(\operatorname{Im} \delta+\operatorname{Im} \Delta) .
$$

To deduce, say, (A) from (C), one omits the last term in (C) and gets $\operatorname{Im} \delta \Delta \supset$ $\operatorname{Im} \delta \cap \operatorname{Ker} \Delta$ whereas the inverse inclusion is obvious. Similarly, (C) follows from (A) and $(B)$ together. 
Assume that $\mathcal{A}$ is finite dimensional over a field and $\delta$ varies in a family, say $\left\{\delta_{a}\right\}$. After a generalization, dimension of $\operatorname{Im} \delta \Delta$ can only jump, and that of Ker $\delta$ only drop. Hence if (B) holds at a point, it holds in an open neighborhood of it. In the case of the Dolbeault complex (cf $[\mathrm{BK}]$ ), only the cohomology will be finite-dimensional. The validity of (C) for a particular $\delta=\delta_{0}$ follows from the Kähler formalism. The argument of the previous section (Theorem 4.2 (ii)) furnishes the same result for the generic formal deformation.

5.5. Integral. Let $(\mathcal{A}, \Delta, \delta)$ be a dGBV-algebra. An even $k$-linear functional $\int: \mathcal{A} \rightarrow k$ is called an integral if the following two conditions are satisfied:

$$
\begin{aligned}
& \forall a, b \in \mathcal{A}, \int(\delta a) b=(-1)^{\tilde{a}+1} \int a \delta b, \\
& \forall a, b \in \mathcal{A}, \int(\Delta a) b=(-1)^{\tilde{a}} \int a \Delta b .
\end{aligned}
$$

Notice that (5.18) is equivalent to $\forall a \in \mathcal{A}, \int \delta a=0$ because $\delta$ is a $k$-derivation. Applying (5.19) to $b=1$, we see that $\forall a \in \mathcal{A}, \int \Delta a=0$ as well.

5.5.1. Proposition. Let $\int$ be an integral for $(\mathcal{A}, \Delta, \delta)$.

(i) If $a$ or $b$ belongs to $\operatorname{Ker} \Delta$, we have

$$
\int \partial_{a} b=\int[a \bullet b]=0
$$

Hence if a satisfies (5.11) and (5.12), $\int$ is an integral for $\left(\mathcal{A}, \Delta, \delta_{a}\right)$ as well.

(ii) $\int$ induces a linear functional on $H(\mathcal{A}, \Delta)$ and $H\left(\mathcal{A}, \delta_{a}\right)$ for all a as above. These functionals are compatible with the identifications following from the condition (C).

Proof. If, say, $\Delta a=0$, we have

$$
\int \partial_{a} b=\int\left((-1)^{\tilde{a}} \Delta(a b)-(-1)^{\tilde{a}}(\Delta a) b-a \Delta b\right)=-\int a \Delta b=-(-1)^{\tilde{a}} \int \Delta a b=0 .
$$

The rest is straightforward.

5.6. Metric. If $\int$ is an integral on $(\mathcal{A}, \Delta, \delta)$, we can define the scalar products on $H\left(\mathcal{A}, \delta_{a}\right)$ induced by the symmetric scalar product $(a, b) \mapsto \int a b$ on $\mathcal{A}$. For the construction of Frobenius manifolds, it is necessary to ensure that these scalar products are non-degenerate.

Integral and metric are compatible with base extensions.

5.7. Additional grading. Assume now that $\mathcal{A}$ as commutative $k$-superalgebra is graded by an additive subgroup of $k$. Thus $\mathcal{A}=\oplus_{n} \mathcal{A}^{n}, k \in \mathcal{A}^{0}, \mathcal{A}^{m} \mathcal{A}^{n} \subset \mathcal{A}^{m+n}$, and each $\mathcal{A}^{i}$ is graded by parity. We write $|a|=i$ if $a \in \mathcal{A}^{i}$. Various induced gradings and degrees of homogeneous operations are denoted in the same way. (In the main example of $[\mathrm{BK}], \mathcal{A}$ is $\mathbf{Z}$-graded, and each $\mathcal{A}^{i}$ is either even, or odd, but this plays no role in general).

All base extensions then must be furnished by the similar grading or its topological completion.

We will assume also that $|\Delta|=-1$. It follows that $|[a \bullet b]|=|a|+|b|-1$ which we interpret as $|\bullet|=-1$. Moreover, we postulate that $|\delta|=1$. This means that the 
shifted differential $\delta_{\gamma}$ can be homogeneous only for $|\gamma|=2$, and similarly for extended base.

Homology space $H$ in all its incarnations (cf. Lemma 5.4.1) inherits the grading from $\mathcal{A}$. The dual space $H^{t}$ is graded in such a way that the pairing $H^{t} \otimes H \rightarrow k$ has degree zero. This induces the additional grading (or more precisely, the notion of homogeneity) on $K=k\left[\left[H^{t}\right]\right]$ (which might be the product rather than the sum of its homogeneous components).

Integral is supposed to have a definite degree, not necessarily zero (and usually non-zero).

5.8. Tensor product of GBV-algebras. Let $\left(\mathcal{A}_{i}, \Delta_{i}\right), i=1,2$, be two GBValgebras over $k$. Put $\mathcal{A}:=\mathcal{A}_{1} \otimes \mathcal{A}_{2}, \Delta:=\Delta_{1} \otimes 1+1 \otimes \Delta_{2}: \mathcal{A} \rightarrow \mathcal{A}$.

5.8.1. Proposition. $(\mathcal{A}, \Delta)$ is a $G B V$-algebra. We have for $a_{i}, b_{i} \in \mathcal{A}_{i}$

$$
\partial_{a_{1} \otimes a_{2}}=\partial_{a_{1}} \otimes(-1)^{\tilde{a}_{2}} l_{a_{2}}+l_{a_{1}} \otimes \partial_{a_{2}}
$$

or equivalently

$$
\left[a_{1} \otimes a_{2} \bullet b_{1} \otimes b_{2}\right]=(-1)^{\tilde{a}_{2}\left(\widetilde{b}_{1}+1\right)}\left[a_{1} \bullet b_{1}\right] \otimes a_{2} b_{2}+(-1)^{\widetilde{b}_{1}\left(\widetilde{a}_{2}+1\right)} a_{1} b_{1} \otimes\left[a_{2} \bullet b_{2}\right] .
$$

Proof. (5.21) is established by a straightforward calculation which we omit. From (5.21) it follows that $\partial_{a_{1} \otimes a_{2}}$ are derivations. Hence $\partial_{a}$ are derivations for all $a \in \mathcal{A}$ so that $(\mathcal{A}, \Delta)$ is a GBV-algebra. (5.22) is a rewriting of (5.21).

Clearly, tensor product is commutative and associative with respect to the standard isomorphisms.

If $\delta_{i}: \mathcal{A}_{i} \rightarrow \mathcal{A}_{i}$ are odd derivations of $\left(\mathcal{A}_{i}, \Delta_{i}\right)$ satisfying (5.7), then $\delta:=\delta_{1} \otimes 1+$ $1 \otimes \delta_{2}$ is an odd derivation of $\mathcal{A}_{1} \otimes \mathcal{A}_{2}$ satisfying (5.7).

If $\mathcal{A}_{i}$ are furnished with additional gradings having the properties postulated above, then the total grading on $\mathcal{A}_{1} \otimes \mathcal{A}_{2}$ satisfies the same conditions.

5.8.2. Decomposable solutions to the Maurer-Cartan equation. In the notation of the previous subsection, let $(\mathcal{A}, \Delta, \delta)$ be the tensor product of $\left(\mathcal{A}_{i}, \Delta_{i}, \delta_{i}\right), i$ $=1,2$. Assume that $a_{i} \in \mathcal{A}_{i}$ satisfy the Maurer-Cartan equation (5.11). Then from (5.22) it follows that $a:=a_{1} \otimes 1+1 \otimes a_{2}$ satisfies (5.11) as well. Moreover, if $\Delta_{i} a_{i}=0$, then $\Delta a=0$, so that $\left(\mathcal{A}, \Delta, \delta_{a}\right)$ is the differential GBV-algebra. Such structures will be called decomposable ones.

5.9. Example: dGBV algebras related to the Calabi-Yau manifolds ([BK]). Let $W$ be a compact complex Kähler manifold with the property $\Omega_{W}^{\max } \cong \mathcal{O}_{W}$ ("weak Calabi-Yau"). Choose once for all a nonzero holomorphic volume form $\Omega$ on $W$. Consider the $\mathbf{C}-$ algebra

$$
\mathcal{A}_{W}:=\bigoplus_{p, q \geq 0} \Gamma_{C^{\infty}}\left(W, \wedge^{q}\left(\bar{T}_{M}^{*}\right) \otimes \wedge^{p}\left(T_{W}\right)\right)
$$

with $\mathbf{Z}_{2}$-grading $(p+q) \bmod 2$.

The map $\gamma \mapsto \gamma \vdash \Omega$ identifies $\mathcal{A}_{W}$ with the complexified de Rham complex of $W$. Let $\Delta$ correspond to $\partial$ with respect to this identification. One can directly check that $\Delta$ satisfies conditions of the first paragraph of 5.1 (Tian-Todorov lemma), hence claims 5.1.1 and 5.1.2 as well. Furthermore, since $\wedge^{*}\left(T_{W}\right)$ is a holomorphic vector 
bundle, $\mathcal{A}_{W}$ can be endowed with the differential $\bar{\partial}$ which we identify with $\delta$. Again, (5.7) can be checked directly so that $\left(\mathcal{A}_{W}, \Delta, \delta\right)$ is a dGBV.

The key property is the validity of Lemma 5.4.1: this is essentially the $\partial \bar{\partial}$-lemma from [DGMS]. Notice that only the existence of Kähler structure on $W$ is needed for its validity, concrete choice does not matter.

The homology space is

$$
H\left(\mathcal{A}_{W}, \delta\right)=H^{*}\left(W, \wedge^{*}\left(T_{W}\right)\right) .
$$

Clearly, using $\Omega$, one can identify it with $H^{*}\left(W, \Omega_{W}^{*}\right)$ as well.

Define the integral by

$$
\int \gamma:=\int_{W}(\gamma \vdash \Omega) \wedge \Omega
$$

It does not vanish only on the component $q=p=\operatorname{dim} W$. Properties (5.18) and (5.19) follow from the Stokes formula.

The algebra $\mathcal{A}_{W}$ possesses the additional $\mathbf{Z}$-grading by $q+p$ satisfying all the conditions of sec. 5.7.

5.10. Example: dGBV algebras related to the symplectic manifolds satisfying the strong Lefschetz condition $([\mathrm{Me}])$. Let now $(U, \omega)$ be a real manifold of dimension $2 m$ endowed with a symplectic form $\omega$. Denote by $\langle$,$\rangle the pairing on$ $\Omega^{*}(U)$ induced by the symplectic form. Put

$$
\left(\mathcal{B}_{U}, \Delta, \delta\right):=\left(\Omega^{*}(U),(-1)^{*+1} \star d \star, d\right)
$$

where $\star: \Omega^{k}(U) \rightarrow \Omega^{2 m-k}(U)$ is the symplectic star operator defined by

$$
\beta \wedge(\star \alpha)=\langle\beta, \alpha\rangle \frac{\omega^{m}}{m !} .
$$

$\mathbf{Z}_{2}$-grading is the degree of the diferential form $\bmod 2$. Calculating $\Delta$ in local coordinates, one sees that it is the differential operator of second order satisfying (5.1), whereas (5.7) follows from (5.27). Thus $\left(\mathcal{B}_{U}, \Delta, \delta\right)$ is a dGBV-algebra.

5.10.1. Proposition. Assume that $(U, \omega)$ satisfies the strong Lefschetz condition, that is, the cup product

$$
\left[\omega^{k}\right] \cup: H^{m-k}(U) \rightarrow H^{m+k}(U)
$$

is an isomorphism for each $k \leq m$. Then $\left(\mathcal{B}_{U}, \Delta, \delta\right)$ satisfies Lemma 5.4.1. [Mat].

$\mathrm{S}$. Merkulov [Me] proves this, completing some earlier results from [Kos], [Br] and

From now on, we will assume that the strong Lefschetz condition holds, so that $U$ is compact. Then we can define the integral on $\mathcal{B}_{U}$ :

$$
\int \gamma:=\int_{U} \gamma
$$

Properties (5.18) and (5.19) follow from the Stokes formula combined with the identities $\star(\star \alpha)=\alpha$ and $\beta \wedge(\star \alpha)=(\star \beta) \wedge \alpha$.

The standard $\mathbf{Z}$-grading of $\Omega^{*}(W)$ then satisfies all conditions of sec. 5.7.

6. From dGBV-algebras to Frobenius manifolds. 
6.1. Normalized formal solution to the master equation. In this section, we fix a dGBV $k$-algebra $(\mathcal{A}, \Delta, \delta)$ and the derived odd bracket [ $\bullet$ ] on it. We will assume that this algebra satisfies a series of assumptions which will be introduced and numbered consecutively.

Assumption 1. $(\mathcal{A}, \Delta, \delta)$ satisfies conditions of the Lemma 5.4.1. Moreover, the homology group $H=H(\mathcal{A}, \delta)$ (and any group naturally isomorphic to it) is a free $k-$ module of finite rank.

Choosing an indexed basis $\left[c_{i}\right], c_{i} \in \mathcal{A}$ of $H$ and the dual basis $\left(x_{i}\right)$ of $H^{t}$ we will always assume that $c_{0}=1$. As in 4.1 , but now conserving parity, we put $K:=$ $k\left[\left[H^{t}\right]\right]=k\left[\left[x_{i}\right]\right]$. We will denote by $X_{i}=\partial / \partial x_{i}$ the respective partial derivatives acting on $K$ and on $K \otimes \mathcal{A}, K \otimes H$ etc via the first factor.

6.1.1. Proposition. If $(\mathcal{A}, \Delta, \delta)$ satisfies Assumption 1 above, then there exists a generic even formal solution $\Gamma=\sum_{i} \Gamma_{i} \in K \otimes \operatorname{Ker} \Delta$ to the master equation

$$
\delta \Gamma+\frac{1}{2}[\Gamma \bullet \Gamma]=0
$$

with the following properties:

(i) $\Gamma_{0}=0, \Gamma_{1}=\sum x_{i} c_{i}, \Gamma_{n} \in K \otimes \operatorname{Im} \Delta$ for all $n \geq 2$. Here $c_{i} \in \operatorname{Ker} \Delta \cap \operatorname{Ker} \delta$, and $\Gamma_{n}$ is the homogeneous component of $\Gamma$ of degree $n$ in $\left(x_{i}\right)$.

(ii) Moreover, this $\Gamma$ can be chosen in such a way that $X_{0} \Gamma=1$.

Such a solution will be called normalized.

Proof. The first statement follows from the Theorem 4.2 (i) applied to the odd differential Lie superalgebra $(\operatorname{Ker} \Delta,[\bullet], \delta)$.

We must only check that the conditions of the applicability of this theorem are satisfied. To facilitate the bookkeeping for the reader, we register the correspondences between the old and the new notation: $\operatorname{Ker} d$ becomes $\operatorname{Ker} \Delta \cap \operatorname{Ker} \delta, \operatorname{Ker} \phi$ turns into $\operatorname{Im} \Delta, \operatorname{Im} d$ corresponds to $\operatorname{Im} \delta \Delta$. All of this forms a part of Lemma 5.4.1.

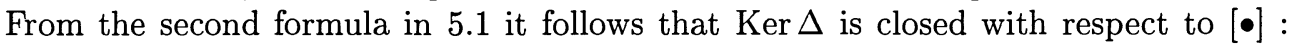
$[a \bullet b]=(-1)^{\tilde{a}} \Delta(a b)$. This formula shows as well that [•] induces zero operation on $H(\operatorname{Ker} \Delta, \delta):$ if $a, b \in \operatorname{Ker} \Delta \cap \operatorname{Ker} \delta$, then $[a \bullet b] \in \operatorname{Im} \Delta \cap \operatorname{Ker} \delta=\operatorname{Im} \delta \Delta$.

It remains to check the assertion (ii). Clearly, our choice $c_{0}=1$ assures that $X_{0} \Gamma_{1}=1$. Assume by induction that $\Gamma_{2}, \ldots, \Gamma_{n}$ do not depend on $x_{0}$. Clearly, $\left[c_{0} \bullet a\right]=0$ for any $a$, so that in the (odd version of the) equation (4.5) the right hand side is independent of $x_{0}$ as well. Since the argument showing the existence of $\Gamma_{n+1}$ in the proof of 4.2 can be applied to each coefficient of the monomials in $x_{i}$ separately, we may find $\Gamma_{n+1}$ independent of $x_{0}$. The final normalization argument can be also applied coefficientwise.

6.2. The (pre)-Frobenius manifold associated to $(\mathcal{A}, \Delta, \delta)$. Consider the formal manifold $M$, the formal spectrum of $K$ over $k$. The flat coordinates will be by definition $\left(x_{i}\right)$ so that the space of flat vector fields can be canonically identified with $H$. We fix a normalized $\Gamma$ as above.

6.2.1. Lemma. The bi-differential group $\left(\mathcal{A}_{K}, \Delta_{K}, \delta_{\Gamma}\right)$ satisfies the conditions and conclusions of Lemma 5.4.1.

Proof. Clearly, $\delta_{\Gamma}^{2}=\Delta_{K}^{2}=\left[\Delta_{K}, \delta_{\Gamma}\right]=0$ (the latter follows from (5.3)). From the Assumption 1 and the proof of 6.6.1 above we see that we can apply the Theorem 4.2 
(ii) to $\left(K \otimes \operatorname{Ker} \Delta, \delta_{\Gamma}\right)$ instead of $\left(g_{K}, d_{K, \Gamma}\right)$. The inclusion (4.6) reads in this context:

$$
\operatorname{Im} \Delta_{K} \cap \operatorname{Ker} \delta_{\Gamma} \subset \operatorname{Im} \delta_{\Gamma} \Delta_{K}
$$

which implies the condition (B).

To check (A), consider the inclusion map

$$
\operatorname{Im} \delta_{\Gamma} \Delta_{K} \rightarrow \operatorname{Im} \delta_{\Gamma} \cap \operatorname{Ker} \Delta_{K}
$$

It becomes an isomorphism after reduction modulo $\left(x_{i}\right)$ in view of the Assumption 1. Hence it is an isomorphism.

6.2.2. o-multiplication on tangent fields. We will define now the $K$-linear o-multiplication on the $K$-module of all vector fields $\mathcal{T}_{M}=K \otimes H=H_{K}$.

To this end we first apply Theorem 4.2 (ii) to the odd differential Lie algebra $\left(\operatorname{Ker} \Delta, \delta_{\Gamma}\right)$. It shows that the homology of this algebra is naturally identified with $H_{K}$.

In view of Lemmas 5.4.1 and 6.2.1 we know that the injection $\left(\operatorname{Ker} \Delta_{K}, \delta_{\Gamma}\right) \rightarrow$ $\left(\mathcal{A}_{K}, \delta_{\Gamma}\right)$ induces isomorphism of homology $H_{K}=\operatorname{Ker} \delta_{\Gamma} / \operatorname{Im} \delta_{\Gamma}$. But $\operatorname{Ker} \delta_{\Gamma}$ is a commutative $K$-subalgebra of $A_{K}$ and $\operatorname{Im} \delta_{\Gamma}$ is an ideal in it. Hence $H_{K}$ inherits the multiplication which we denote $\circ$. We record the following "explicit" formula for it. Interpreting any $X \in H_{K}$ as the derivation $\bar{X}$ of $K \otimes \mathcal{A}$ acting through the first factor (cf. Corollary 4.2.1), we have:

$$
\overline{X \circ Y} \Gamma \equiv \bar{X} \Gamma \cdot \bar{Y} \Gamma \bmod \operatorname{Im} \delta_{\Gamma}
$$

(dot here means the associative multiplication in $\mathcal{A}_{K}$ ). This follows directly from the Corollary 4.2.1 applied to our situation. Notice that whereas $\bar{X} \Gamma$ and $\bar{Y} \Gamma$ lie in $\operatorname{Ker} \Delta_{K} \cap \operatorname{Ker} \delta_{\Gamma}$, their product generally lies only in the larger group $\operatorname{Ker} \delta_{\Gamma}$.

Directly from the initial definition one sees that $e:=X_{0}$ is the flat identity for $\circ$.

In order to complete the description of the pre-Frobenius structure, it remains to choose a flat metric on $M$.

ASSUMPTION 2. There exists an integral $\int$ for $(\mathcal{A}, \Delta, \delta)$ such that the bilinear form on $H=H(A, \delta)$ induced by $(X, Y) \mapsto \int \bar{X} \Gamma \cdot \bar{Y} \Gamma$ is non-degenerate.

Denoting this form $g$ we clearly have the invariance property defining the symmetric multiplication tensor $A$ :

$$
g(X, Y \circ Z)=g(X \circ Y, Z):=A(X, Y, Z) .
$$

We will check now that this structure is actually Frobenius. Since the o-multiplication is associative, we have only to establish its potentiality.

To this end we will check Dubrovin's criterium: the structure connection $\nabla_{\lambda}$ on $\mathcal{T}_{M}$ is flat (cf. [M], Ch. I, Theorem 1.5).

To be more precise, let $\nabla_{0}$ be the flat connection on $\mathcal{T}_{M}$ whose horizontal sections are $H$. Clearly, $\nabla_{0, Y}(Z)=\bar{Y}(Z)$ where this time $\bar{Y}$ means $Y$ acting on $K \otimes H$ via $K$. By definition,

$$
\nabla_{\lambda, Y}(Z)=\bar{Y}(Z)+\lambda Y \circ Z
$$

where $\lambda$ is an even parameter.

We have the canonical surjection Ker $\Delta_{K} \rightarrow H_{K}$ and the two lifts of $X$ both denoted by $\bar{X}$ are compatible with this surjection, and also with embedding $\operatorname{Ker} \Delta_{K} \subset$ 
$\mathcal{A}_{K}$. Therefore the section of $\operatorname{Ker} \Delta_{K} \rightarrow H_{K}$ denoted $\psi$ in the Corollary 4.2.1 sends $\bar{Y}(Z)$ to $\bar{Y}(\bar{Z} \Gamma)$, and $\nabla_{\lambda, Y}(Z)$ lifts to $\bar{Y}(\bar{Z} \Gamma)+\lambda \bar{Y} \Gamma \cdot \bar{Z} \Gamma$ in view of (6.1) and (6.3).

Our preparations being now completed, we can prove

6.2.3. Theorem. The connection $\nabla_{\lambda}$ is flat. Hence the pre-Frobenius structure defined above is potential.

Proof. Applying (6.3) twice, we find

$$
\nabla_{\lambda, X} \nabla_{\lambda, Y}(Z)=\bar{X}(\bar{Y}(Z))+\lambda \bar{X}(Y \circ Z)+\lambda X \circ \bar{Y}(Z)+\lambda^{2} X \circ Y \circ Z .
$$

We may and will consider only the case when $X, Y$ supercommute (e.g. $X, Y \in H$ ). In order to establish flatness, it suffices to check that

$$
\bar{X}(Y \circ Z)+X \circ \bar{Y}(Z)=(-1)^{\tilde{X}} \tilde{Y}(\bar{Y}(X \circ Z)+Y \circ \bar{X}(Z)) .
$$

We will see that already the $\psi$-lifts of both sides of (6.5) coincide up to $\operatorname{Im} \delta_{\Gamma}$. In fact, $\bar{X}(Y \circ Z)$ lifts to $\bar{X}(\bar{Y} \Gamma \cdot \bar{Z} \Gamma), X \circ \bar{Y}(Z)$ lifts to $\bar{X} \Gamma \cdot \bar{Y}(\bar{Z} \Gamma)$ so that (6.5) becomes

$$
\begin{gathered}
\bar{X}(\bar{Y} \Gamma) \cdot \bar{Z} \Gamma+(-1)^{\tilde{X}} \tilde{Y} \bar{Y} \Gamma \cdot \bar{X}(\bar{Z} \Gamma)+\bar{X} \Gamma \cdot \bar{Y}(\bar{Z} \Gamma)= \\
(-1)^{\tilde{X}} \tilde{Y} \bar{Y}(\bar{X} \Gamma) \cdot \bar{Z} \Gamma+\bar{X} \Gamma \cdot \bar{Y}(\bar{Z} \Gamma)+(-1)^{\tilde{X}} \tilde{Y} \bar{Y} \Gamma \cdot \bar{X}(\bar{Z} \Gamma) .
\end{gathered}
$$

This finishes the proof.

6.3. Euler field. Assume now that $\mathcal{A}$ is endowed with a grading satisfying the conditions of 5.7. All the previous discussion makes sense, and the results hold true, if we add appropriate grading conditions at certain places, the most important of which is $|\Gamma|=2$ implying $|\circ|=2$ in view of (6.1).

Denote by $E$ the derivation of $K$ defined by the following Euler condition:

$$
\forall f \in K, E f=\frac{1}{2}|f| f
$$

where || is the grading induced on $H^{t}$ from $\mathcal{A}$ via $H$. In cooordinates as in 6.1 we have

$$
E=\frac{1}{2} \sum_{i}\left|x_{i}\right| x_{i} X_{i} .
$$

Assumption 3. Assume that the integral is homogeneous and denote its degree by $2 D-4$.

For the general discussion of spectrum cf. [M], Chapter I, §2, cf. also 1.3 above for spectrum of quantum cohomology.

6.3.1. Proposition. $E$ is an Euler field on the formal Frobenius manifold described in 6.2. Its spectrum is $\left(D ; d_{i}\right.$ with multiplicity $\left.\operatorname{dim} H^{-2 d_{i}}\right)$, and $d_{0}=1$.

Proof. Comparing (6.7) with the notation of $[\mathrm{M}]$, we see that the spectrum of $-\operatorname{ad} E$ on $H=\mathcal{T}_{M}^{f}$ is $d_{i}$ with multiplicity $\operatorname{dim} H^{-2 d_{i}}$ where

$$
d_{i}=\frac{1}{2}\left|x_{i}\right|=-\frac{1}{2}\left|X_{i}\right|
$$

Since $X_{0} \circ X=X$ and $|\circ|=2$, we have $d_{0}=1$.

We must now check the formula

$$
E(g(X, Y))-g([E, X], Y)-g(X,[E, Y])=D g(X, Y) .
$$


It suffices to do this for the case when $X, Y$ are flat vector fields having definite degrees. Then $[E, X]=\frac{|X|}{2} X$. Since $g(X, Y) \in k,(6.9)$ becomes

$$
(|X|+|Y|+2 D) g(X, Y)=0 \text {. }
$$

But $g(X, Y)=\int \bar{X} \Gamma \cdot \bar{Y} \Gamma$ vanishes unless $2 D-4+|X|+2+|Y|+2=0$ which proves (6.9).

Furthermore, from (6.1) we infer that $|X \circ Y|=|X|+|Y|+2$. Hence if $X_{i} \circ X_{j}=$ $\sum_{k} A_{i j}{ }^{k} X_{k}$, we have

$$
E A_{i j}{ }^{k}=\frac{1}{2}\left|A_{i j}{ }^{k}\right| \cdot A_{i j}{ }^{k}=\frac{1}{2}\left(\left|X_{i}\right|+\left|X_{j}\right|-\left|X_{k}\right|+2\right) A_{i j}{ }^{k} .
$$

Comparing this with the formula (2.18) of [M], Chapter I and taking into account (6.8), we see that $E$ satisfies the Definition 2.2.1 of [M], loc. cit. This finishes the proof.

Notice that the Euler field (6.7) contains no flat summand: $X_{i}$ with $d_{i}=0$ do not contribute. Hence if this construction furnishes a Frobenius manifold which is quantum cohomology of some $V$, then $c_{1}(V)$ must vanish (modulo torsion).

6.3.2. Remark. Comparing the Frobenius manifold produced from a dGBValgebra with grading as above to a quantum cohomology Frobenius manifold, one must first shift the dGBV-grading by two. Then $X_{0}$ and $\circ$ acquire the degree zero.

6.4. Explicit potential. The direct way to establish potentiality is to find an even series $\Phi \in K$ such that for all $X, Y, Z \in H$ we have $A(X, Y, Z)=X Y Z \Phi$ (from now on, we write $X$ instead of $\bar{X}$ in order to denote derivations on various $K$-modules acting through $K$ ). Moreover, it suffices to check this for $X=Y=Z$. We will give here the beautiful formula of Chern-Simons type for $\Phi$ discovered in [BK].

Extend the integral to the $K$-linear map $\int: \mathcal{A}_{K} \rightarrow K$. For a fixed normalized $\Gamma$ put $\Gamma=\Gamma_{1}+\Delta B$ where $B_{0}=B_{1}=0$ and $\Delta$ means $\Delta_{K}$.

\subsubsection{TheOREM. The formal function}

$$
\Phi:=\int\left(\frac{1}{6} \Gamma^{3}-\frac{1}{2} \delta B \Delta B\right) .
$$

is a potential for the Frobenius manifold defined above.

Proof. We have to prove that for any $X \in H$

$$
A(X, X, X)=\int(X \Gamma)^{3}=X^{3} \Phi .
$$

We supply below the detailed calculation consisting of the series of elementary steps, each being an application of one of the identities (5.18), (5.19), Leibniz rule for (super)derivations and the fact that $\delta, \Delta, X$ pairwise supercommute. Moreover, we use the master equation in the form $\Delta \Gamma^{2}=-2 \delta \Gamma$ following from $\Delta \Gamma=0$. Finally, $\delta \Gamma_{1}=X^{n} \Gamma_{1}=0$ for $n \geq 2$ so that $\delta \Gamma=\delta \Delta B, X^{n} \Gamma=X^{n} \Delta B$.

We start with treating the first summand of the right hand side of (6.10). The derivation $X$ is interchangeable with integration, so we have by the Leibniz rule

$$
X^{3}\left(\frac{1}{6} \int \Gamma^{3}\right)=\int(X \Gamma)^{3}+\int\left(\left(2+(-1)^{\tilde{X}}\right) \Gamma \cdot X \Gamma \cdot X^{2} \Gamma+\frac{1}{2} \Gamma^{2} \cdot X^{3} \Gamma\right) .
$$


The second summand of (6.10) is added in order to cancel the extra terms in (6.12).

First, we rewrite it:

$$
\frac{1}{2} \int \delta B \Delta B=\frac{1}{2} \int B \delta \Delta B=\frac{1}{2} \int B \delta \Gamma=-\frac{1}{4} \int B \Delta\left(\Gamma^{2}\right)=\frac{1}{4} \int \Delta B \cdot \Gamma^{2} .
$$

(We could have chosen the last expression in (6.13) from the start).

Now, again by Leibniz rule,

$$
\begin{gathered}
\frac{1}{4} X^{3} \int \Delta B \cdot \Gamma^{2}=\frac{1}{4} \int\left(X^{3}(\Delta B) \Gamma^{2}+\left(2+(-1)^{\tilde{X}}\right) X^{2}(\Delta B) \cdot X\left(\Gamma^{2}\right)\right. \\
\left.+\left(2+(-1)^{\tilde{X}}\right) X(\Delta B) \cdot X^{2}\left(\Gamma^{2}\right)+\Delta B \cdot X^{3}\left(\Gamma^{2}\right)\right)
\end{gathered}
$$

The first two summands in (6.14) can be directly rewritten in the same form as in (6.12):

$$
\begin{gathered}
X^{3}(\Delta B) \cdot \Gamma^{2}+\left(2+(-1)^{\tilde{X}}\right) X^{2}(\Delta B) \cdot X\left(\Gamma^{2}\right)= \\
X^{3} \Gamma \cdot \Gamma^{2}+\left(2+(-1)^{\tilde{X}}\right) X^{2} \Gamma \cdot X\left(\Gamma^{2}\right) .
\end{gathered}
$$

The third summand takes somewhat more work:

$$
\begin{gathered}
\int X(\Delta B) \cdot X^{2}\left(\Gamma^{2}\right)=-\int X B \cdot X^{2}\left(\Delta \Gamma^{2}\right)=2 \int X B \cdot X^{2}(\delta \Gamma)= \\
2 \int X B \cdot \delta\left(X^{2} \Gamma\right)=2 \int X B \cdot \delta \Delta\left(X^{2} B\right)=-2 \int \delta \Delta X B \cdot X^{2} B= \\
-2 \int X \delta \Gamma \cdot X^{2} B=\int X \Delta\left(\Gamma^{2}\right) \cdot X^{2} B=\int X\left(\Gamma^{2}\right) \cdot X^{2} \Delta B= \\
\int X\left(\Gamma^{2}\right) \cdot X^{2} \Gamma .
\end{gathered}
$$

Finally, the fourth summand is calculated similarly, but in two steps. We start with an expression of the second order in $X$ :

$$
\begin{gathered}
\int \Delta B \cdot X^{2}\left(\Gamma^{2}\right)=-\int B \cdot X^{2}\left(\Delta \Gamma^{2}\right)=2 \int B \cdot X^{2}(\delta \Gamma)= \\
2 \int B \cdot \delta\left(X^{2} \Gamma\right)=2 \int B \cdot \delta \Delta\left(X^{2} B\right)=-2 \int \delta \Delta B \cdot X^{2} B= \\
-2 \int \delta \Gamma \cdot X^{2} B=\int \Delta\left(\Gamma^{2}\right) \cdot X^{2} B=\int \Gamma^{2} \cdot X^{2} \Delta B= \\
\int \Gamma^{2} \cdot X^{2} \Gamma .
\end{gathered}
$$

Apply now $X$ to the first and the last expressions of (6.17). We get

$$
\int X(\Delta B) \cdot X^{2}\left(\Gamma^{2}\right)+\int \Delta B \cdot X^{3}\left(\Gamma^{2}\right)=\int X\left(\Gamma^{2}\right) \cdot X^{2} \Gamma+\int \Gamma^{2} \cdot X^{3} \Gamma
$$


Comparing this with (6.16), one gets

$$
\int \Delta B \cdot X^{3}\left(\Gamma^{2}\right)=\int \Gamma^{2} \cdot X^{3} \Gamma
$$

Putting all of this together, one obtains finally (6.11).

6.5. Example: Frobenius manifolds of B-type, related to the CalabiYau manifolds. Returning now to the examples of 5.9, one sees that all assumptions of this section hold so that we get a class of Frobenius manifolds, which we may call BK-models of Calabi-Yau manifolds $W$. In particular, we can easily calculate the $d$-spectrum which is:

$$
\left(w ; d \text { with multiplicity } \sum_{q+p=2 d} h^{p, w-q}(W)\right), w:=\operatorname{dim}_{\mathbf{C}}(W) .
$$

6.6. Example: Frobenius manifolds related to the symplectic manifolds satisfying the strong Lefschetz condition. Similarly, in the situation of 5.10 we obtain the Frobenius manifold with the $d$-spectrum

$$
\left(m ; d \text { with multiplicity } \operatorname{dim} H^{2 d}(W)\right) .
$$

Notice that in this case as well the anticanonical component of the Euler field vanishes.

It would be interesting to establish isomorphisms between these examples and to understand when they furnish Frobenius manifolds of qc-type. Notice that if $W, U$ are mirror dual Calabi-Yau manifolds, then the spectra of $\mathcal{A}_{W}$ and $\mathcal{B}_{U}$ coincide.

\section{REFERENCES}

[AGV] V. Arnold, S. Gusein-Zade, and A. Varchenko, Singularities of Differentiable Maps, Vols. I, II., Birkhäuser, Boston, 1985 and 1988.

[BK] S. BARANNIKov AND M. KontSEvich, Frobenius manifolds and formality of Lie algebras of polyvector fields, Preprint alg-geom/97010072.

[B] K. BEHREND, Gromov-Witten invariants in algebraic geometry, Inv. Math., 127 (1997), pp. 601-617.

[BF] K. Behrend AND B. Fantechi, The intrinsic normal cone, Inv. Math., 128 (1997), pp. 4588.

[BM] K. BEHREND AND YU. MANin, Stacks of stable maps and Gromov-Witten invariants, Duke Math. J., 85:1 (1996), pp. 1-60.

[Br] J.-L. Brylinski, A differential complex for Poisson manifolds, J. Diff. Geom., 28 (1988), pp. 93-114.

[C] S. Cесотті, $N=2$ Landau-Ginzburg vs. Calabi-Yau $\sigma$-models: non-perturbative aspects, Int. J. of Mod. Phys. A, 6:10 (1991), pp. 1749-1813.

[DGMS] P. Deligne, Ph. Griffiths, J. Morgan, and D. Sullivan, Real homotopy theory of Kähler manifolds, Inv. Math., 29 (1975), pp. 245-274.

[D] B. Dubrovin, Geometry of $2 D$ topological field theories, in Springer LNM, 1620, 1996, pp. $120-348$.

[DZh] B. Dubrovin ANd Youjin Zhang, Extended affine Weyl groups and Frobenius manifolds, Preprint SISSA 67/96/FM.

[Ge1] D. GEPNER, On the spectrum of $2 D$ conformal field theory, Nucl. Phys., B287 (1987), pp. 111-126.

[Ge2] D. GePnER, Fusion rings and geometry, Comm. Math. Phys., 141 (1991), pp. 381-411.

[Ger] M. Gerstenhaber, The cohomology structure of an associative ring, Ann. of Math., 78:2 (1963), pp. 267-288.

[Giv] A. Givental, Equivariant Gromov-Witten invariants, Int. Math. Res. Notes, 13 (1996), pp. 613-663. 
[GoM] W. Goldman and J. Millson, The deformation theory of representations of fundamental groups of compact Kähler manifolds, Publ. Math. IHES, 86 (1988), pp. 43-96.

[Gr] B. Green, Constructing mirror manifolds, in Mirror Symmetry II, B. Greene and S. T. Yau, ed., AMS-International Press, 1996, pp. 29-69.

[G-ZV] S. Gusein-ZADE AND A. VARChEnKo, Verlinde algebras and the intersection form on vanishing cycles, Selecta Math., New. Ser., 3 (1997), pp. 79-97.

[K] R. Kaufmann, The geometry of moduli spaces of pointed curves, the tensor product in the theory of Frobenius manifolds, and the explicit Künneth formula in quantum cohomology, Ph. D. thesis, MPI für Mathematik, Bonn, 1997.

[Ko] M. KontSEvich, Enumeration of rational curves via torus actions, in The Moduli Space of Curves, R. Dijkgraaf, C. Faber, G. van der Geer, ed., Progress in Math., 129, Birkhäuser, 1995, pp. 335-368.

[KM1] M. Kontsevich And Yu. Manin, Gromov-Witten classes, quantum cohomology, and enumerative geometry, Comm. Math. Phys., 164:3 (1994), pp. 525-562.

[KM2] M. Kontsevich AND Yu. MANin, Relations between the correlators of the topological sigma-model coupled to gravity, Preprint alg-geom/970824.

[KMK] M. KontSEvich AND YU. MANin, Quantum cohomology of a product (with Ap:ondix by R. Kaufmann), Inv. Math., 124, f. 1-3 (1996), pp. 313-339.

[Kos] J.-L. Koszul, Crochet de Schouten-Nijenhuis et cohomologir, in Elie Cartan et les Mathématiques D'aujourd'huis, Astérisque, 1985, pp. 251-271.

[LLY] B. H. Lian, K. Liu And S.-T. Yau, Mirror Principle I, alg-geom/9712011.

[LZ] B. H. Lian and G. ZuCKerman, New perspectives on the BRST-algebraic structure of string theory, Comm. Math. Phys., 154 (1993), pp. 613-646, hep-th/9211072.

[M] Yu. Manin, Preprint MPI, 1996, pp. 96-113.

[MM] Yu. Manin and S. Merkulov, Semisimple Frobenius (super)manifolds and quantum cohomology of $\mathbf{P}^{r}$, Topological Methods in Nonlinear Analysis, 9:1 (1997), pp. 107-161, alg-geom/9702014.

[Mat] O. Mathieu, Harmonic cohomology classes of symplectic manifolds, Comm. Math. Helvetici, 70 (1995), pp. 1-9.

[Me] S. Merkulov, Formality of canonical symplectic complexes and Frobenius manifolds, Preprint math/9805072.

[O] T. ODA, K. Saito's period map for holomorphic functions with isolated critical points, in Adv. Studies in Pure Math., 10 (1987), Algebraic Geometry, Sendai, 1985, pp. 591-648.

[S1] K. SAITo, Primitive forms for a universal unfolding of a function with an isolated critical point, Journ. Fac. Sci. Univ. Tokyo, Sec. IA, 28:3 (1982), pp. 775-792.

[S2] K. SAITo, Period mapping associated to a primitive form, Publ. RIMS, Kyoto Univ., 19 (1983), pp. 1231-1264.

[St] J. StAShefF, Deformation theory and the Batalin-Vilkovisky master equation, in Deformation Theory and Symplectic Geometry, D. Sternheimer, et. al., ed., Kluwer, 1997, pp. 271-284.

[Z] J.-B. Zuber, Graphs and reflection groups, Comm. Math. Phys., 179 (1996), pp. 265-294. 